\title{
Synthesis and application of azolium ionic liquid tagged TADDOL catalysts
}

\author{
Zekarias Yacob $^{\mathrm{a}}$ and Jürgen Liebscher ${ }^{\mathrm{a}, \mathrm{b}^{*}}$ \\ ${ }^{a}$ National Institute of Research and Development for Isotopic and Molecular Technologies \\ (INCDTIM), Donath Str. 65-103, RO-400293 Cluj-Napoca, Romania \\ ${ }^{b}$ Institute of Chemistry, Humboldt-University Berlin, D-12489 Berlin, Germany \\ E-mail: liebscher@chemie.hu-berlin.de
}

Dedicated to Prof. Dr. Rainer Beckert on the occasion of his $60^{\text {th }}$ birthday

DOI: http://dx.doi.org/10.3998/ark.5550190.0013.322

\begin{abstract}
Ionic liquid (IL) tagged organocatalysts based on TADDOL $\left(\alpha, \alpha, \alpha^{\prime}, \alpha^{\prime}\right.$-tetraaryl-2,2-dimethyl1,3-dioxolane-4,5-dimethanol) comprising various aryl substituents tethered with triazolium and imidazolium based ionic liquids are synthesised in good yields. Investigation of these IL-tagged catalysts on hydrogen-bonding catalyzed hetero-Diels-Alder (HDA) reactions between activated dienes (such as Rawal's diene and Brassard's diene) and benzaldehyde indicated their potential and limitations for applications in organocatalysis.
\end{abstract}

\section{Introduction}

Seebach et al. introduced TADDOLs ( $\alpha, \alpha, \alpha^{\prime}, \alpha^{\prime}$-tetraaryl-2,2-dimethyl-1,3-dioxolane-4,5dimethanol) 1 (Figure 1) as bidentate alkoxide ligands and chiral auxiliaries in $1983 .{ }^{1}$ Since then, TADDOLs have been successfully utilized as chiral scaffolds in organic synthesis. ${ }^{2}$ Various methodologies have been implemented for the immobilization of TADDOL catalysts with the aim of improving their recyclability and reuse as well as their efficiency and stability mainly as ligands for metal catalysis. ${ }^{3-5}$ TADDOL catalysts immobilized on solid supports can exhibit drawbacks such as low catalyst loading, mass transfer dependent diffusion, low mechanical strength, diminished selectivity and loss of catalytic activity during immobilization. There has been much effort to circumvent these drawbacks, which are often considered inherent to the immobilization of catalysts with solid supports.

Ionic liquids (IL) have shown interesting ability as phase tags for organocatalysts. ${ }^{6-15}$ Ionic liquid tagged catalysts can be tuned to attain a required physical property, such as solubility, or compatibility with the reaction medium and thermal stability. ${ }^{16}$ In this way improved recovery 
and recycling and sometimes even better catalytic activity can be attained. ${ }^{17-19,14}$ Accordingly, we envisaged that a properly functionalized ionic liquid tag can render TADDOLs novel properties and improve their usefulness as organocatalysts. Immobilizing TADDOL catalysts with ionic liquids has the potential to render the TADDOLs tuneable solubility for working under homogeneous conditions, without diminishing their catalytic activity or their amount of catalyst loading, unlike TADDOLs immobilized on solid supports. There have not been reports about investigations of the advantages of ionic liquid tags in immobilizing TADDOL catalysts. Here we report the results of our investigations on the use of 1,2,3-triazolium salts and imidazolium salts as suitable ionic liquid tags for TADDOLs (Figure 1, formula 2).

While imidazolium salts have been fully established as ionic liquids, 1,2,3-triazolium salts were unknown in this areas until recently. We and later on others have developed 1,2,3triazolium salts as versatile IL structures which have found various application as ILs in organocatalysis. ${ }^{20-22,13,9}$ They are easily accessible by $\mathrm{Cu}$-catalyzed cycloaddition of azides and terminal alkynes (Meldal-Sharpless click reaction) ${ }^{23}$ followed by $\mathrm{N}$-alkylation of the resulting 1,2,3-triazole. ${ }^{20,24}$ Since functionality can be introduced virtually by all substituents found in the reactants this synthesis renders very versatile. In addition, the counterion can be easily changed for further tuning of the ionic liquid properties.

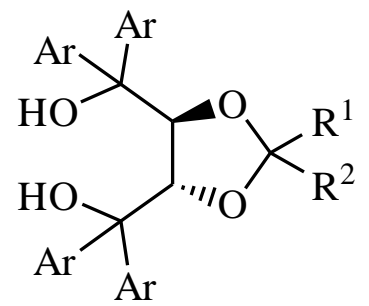

1

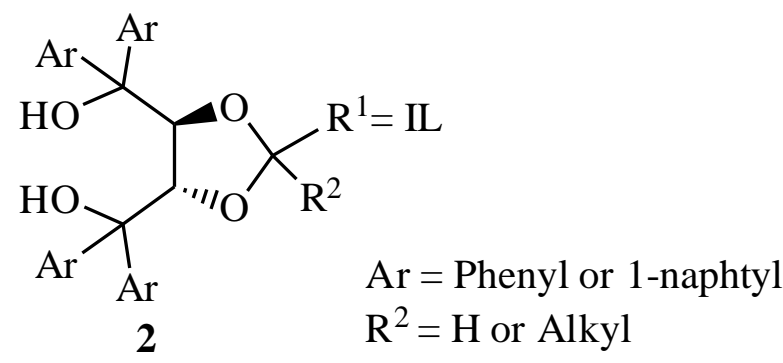

Figure 1. Structure of TADDOL catalysts and envisaged IL-tagging (IL = 1,2,3-triazolium or imidazolium).

\section{Result and Discussion}

TADDOLs are often synthesized starting from dialkyl tartrate and a ketone or an aldehyde forming a 1,4-dioxolane with two ester functions, which are eventually transformed into diarylcarbinols by reaction with Grignard reagents. ${ }^{2}$ In principle, ionic liquid tags could be tethered either to the aryl groups of the carbinol substructures or to the substituents in position 2 of the dioxolane ring. ${ }^{25}$ We chose the latter possibility for its straightforwardness. In order to have much structural flexibility we established the TADDOL moiety at an early stage and various IL-tags were linked at a later stage by means of a propargyl group enabling Cu-catalyzed Meldal-Sharpless click reaction. Thus propargylated 3-hydroxybenzaldehyde $\mathbf{3}^{26,27}$ was 
condensed with dimethyl $L-(+)$-tartate 4 in the presence of trimethyl orthoformate and catalytic amounts of $p$-toluene sulfonic acid to furnish the intended dioxolane ring system $\mathbf{5}$ in good yield. Consecutively Grignard reaction (8 equivalents of Grignard reagent 6) was employed to introduce the necessary aromatic sub-units into the 3-propargyloxyisomer $\mathbf{5}$ smoothly to furnish the envisaged tetraaryl substituted products 7 (Scheme 1).

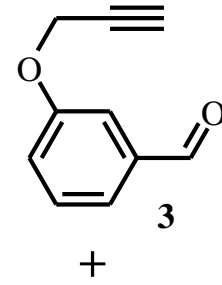<smiles>COC(=O)[C@H](O)[C@@H](O)C(=O)OC</smiles><smiles>C#CCOc1cccc(C2O[C@H](C([Al])([Al])[Al])[C@H](C(O)([Al])[Al])O2)c1</smiles>

$7 \mathbf{a}(\mathrm{Ar}=\mathrm{Ph})$

7b $\left(\mathrm{Ar}=3,5-\mathrm{Me}_{2} \mathrm{C}_{6} \mathrm{H}_{3}\right)$

7c $($ Ar $=1$-naphthyl $)$
p-TsOH (3.7 mol \%)

$\mathrm{HC}\left(\mathrm{OCH}_{3}\right)_{3}$ (1.1 equiv.)

benzene

$0{ }^{\circ} \mathrm{C}$ to reflux

$84 \%$

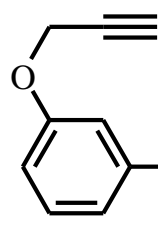

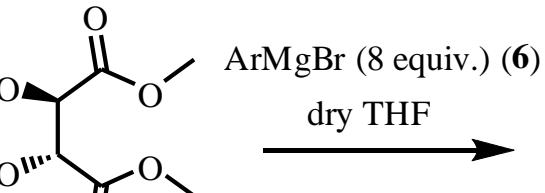

$45-63 \%$<smiles>CCC(C)=O</smiles>

$$
\mathrm{CuSO}_{4}(25 \mathrm{~mol} \%)
$$

sodium ascorbate $(20 \mathrm{~mol} \%)$

8

$\mathrm{MeO}: \mathrm{H}_{2} \mathrm{O}$

$8: 2$

3 days

$82-94 \%$

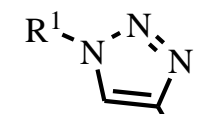

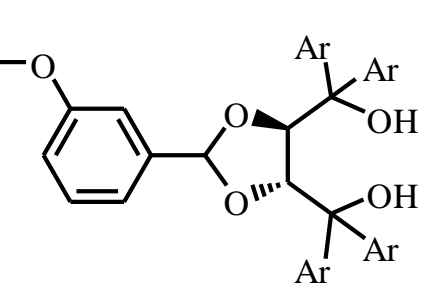

9a $\quad\left(\mathrm{R}^{1}=n-\mathrm{Bu}, \mathrm{Ar}=\mathrm{Ph}\right)$

9b $\left(\mathrm{R}^{1}=n-\mathrm{Bu}, \mathrm{Ar}=3,5-\mathrm{Me}_{2} \mathrm{C}_{6} \mathrm{H}_{3}\right)$

9c $\left(\mathrm{R}^{1}=\mathrm{Bn}, \mathrm{Ar}=1\right.$-naphthyl)

9d $\left(\mathrm{R}^{1}=n-\mathrm{Bu}, \mathrm{Ar}=1\right.$-naphthyl)

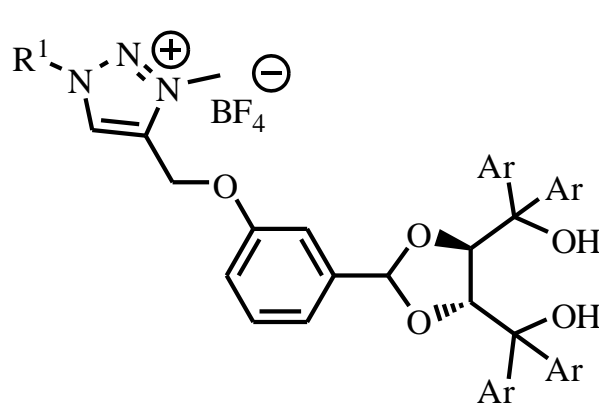

11a $\left(\mathrm{R}^{1}=n-\mathrm{Bu}, \mathrm{Ar}=\mathrm{Ph}\right)$

$11 \mathrm{~b}\left(\mathrm{R}^{1}=n-\mathrm{Bu}, \mathrm{Ar}=3,5-\mathrm{Me}_{2} \mathrm{C}_{6} \mathrm{H}_{3}\right)$

11c $\left(\mathrm{R}^{1}=n-\mathrm{Bu}, \mathrm{Ar}=1\right.$-naphthyl $)$

11d $\left(\mathrm{R}^{1}=\mathrm{Bn}, \mathrm{Ar}=1\right.$-naphthyl $)$
$\mathrm{CH}_{3} \mathrm{I}$ (10 equiv.) $\mathrm{CH}_{3} \mathrm{CN}$, reflux $94-99 \%$

$\mathrm{AgBF}_{4}$
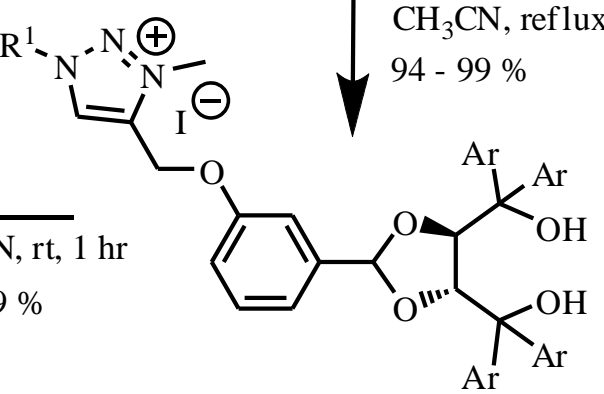

10a $\left(\mathrm{R}^{1}=n-\mathrm{Bu}, \mathrm{Ar}=\mathrm{Ph}\right)$

$10 \mathrm{~b}\left(\mathrm{R}^{1}=n-\mathrm{Bu}, \mathrm{Ar}=3,5-\mathrm{Me}_{2} \mathrm{C}_{6} \mathrm{H}_{3}\right)$

10c $\left(\mathrm{R}^{1}=n-\mathrm{Bu}, \mathrm{Ar}=1\right.$-naphthyl $)$

10d $\left(\mathrm{R}^{1}=\mathrm{Bn}, \mathrm{Ar}=1\right.$-naphthyl $)$

Scheme 1. Synthesis of 1,2,3-triazolium ionic liquid tagged TADDOL catalysts $\mathbf{1 0}$ and 11. 
The isomeric product of $\mathbf{5}$ which was obtained from 4-hydroxybenzaldehyde was found to be unstable and difficult to handle at the later stages of the synthesis. It furnished uncomfortably low yields in the consecutive steps. Seemingly, the electron donating property of the propargyloxy group at position 4 increased the ability to stabilize positive charges at the benzylidene position and thus renders the dioxolane ring more sensitive to ring opening under the influence of acids or Lewis acids, a phenomenon which was reported in the literature. ${ }^{28}$

Now the floor was laid for $\mathrm{Cu}$ (I)-catalyzed click reaction with azides. We chose butyl or benzyl azide 8 and used the well-established click reaction method utilizing $\mathrm{Cu}$ (II)-sulphate (25 mol\%) in the presence of Na-ascorbate $(20 \mathrm{~mol} \%)$ in DMF:methanol (1:1) mixture. The 1,2,3triazoles 9 were obtained in excellent yields. The transformation into the corresponding 1,2,3triazolium iodides $\mathbf{1 0}$ was achieved in high yields by a straight forward alkylation using excess of methyl iodide (10 equivalents) in acetonitrile. Reactions attempted with other methylating agents such as methyl triflate (MeOTf) at room temperature and at $-25{ }^{\circ} \mathrm{C}$ furnished very low yields. Nevertheless, exchange of iodide by other anions was possible by salt metathesis, such as with silver tetrafluoroborate leading to 1,2,3-triazolium tetrafluoroborates $\mathbf{1 1}$ in quantitative yields.

In order to attain imidazolium-tagged TADDOL catalysts we started with 4-(imidazole-1-yl)butanone 12, which was reported as the Michael addition product of imidazole to methyl vinyl ketone in the presence of catalytic amounts of N-methylimidazole in DMSO (Scheme 2). ${ }^{29}$ Unlike in the reported procedures we used triethylamine base. The consecutive use of trimethyl orthoformate procedure previously applied for the synthesis of the analogous1,3-dioxolane $\mathbf{6}$ did not provide the desired product for ketone 12. This is presumably due to the low reactivity of the aliphatic ketone 12 as compared to the aldehyde $\mathbf{6}$ under this reaction condition. The 1,3dioxolane 13 was prepared in good yield by reaction of 12 with dimethyl tartrate in the presence of the Lewis acid boron trifluoride etherate in ethyl acetate. The subsequent Grignard reaction with phenyl magnesium bromide (10 fold excess) provided the carbinol 14 in $58 \%$ yield. NMethylation with methyl iodide (5 equivalents) to imidazolium salt 15 and further salt metathesis to the tetrafluoroborate $\mathbf{1 6}$ with silver tetrafluoroborate took place in excellent yields.

With these IL-tagged TADDOLs in hand we approached their application as IL-tagged organocatalysts. We report here our first attempts to use them in hetero-Diels -Alder reactions (HDA reaction) as potential recyclable catalysts. The IL-tagged TADDOL catalysts (10, 11, 15 and 16) were first investigated in the hydrogen-bonding promoted HDA reaction of Brassard's diene 17 with benzaldehyde (Table 1). Initially, the reaction was carried out at room temperature using 2.5 - 5.0 molar equivalent of freshly distilled benzaldehyde in the presence of $20 \mathrm{~mol} \%$ of catalyst. The catalysts constitute ionic liquid subunits, which have limited solubility in non-polar solvents. However, they are completely soluble in excess of benzaldehyde, which serves as a solvent for the neat reactions. Under these conditions the intended cycloadducts 18 were obtained, however, as racemates with low to moderate yields (Table 1). The same reaction was reported by K. Ding et al, ${ }^{30}$ for non-supported naphthyl TADDOLs affording $67 \%$ yield and $83 \%$ ee. A cycloaddition product was not reported when the TADDOL catalyst was missing. 


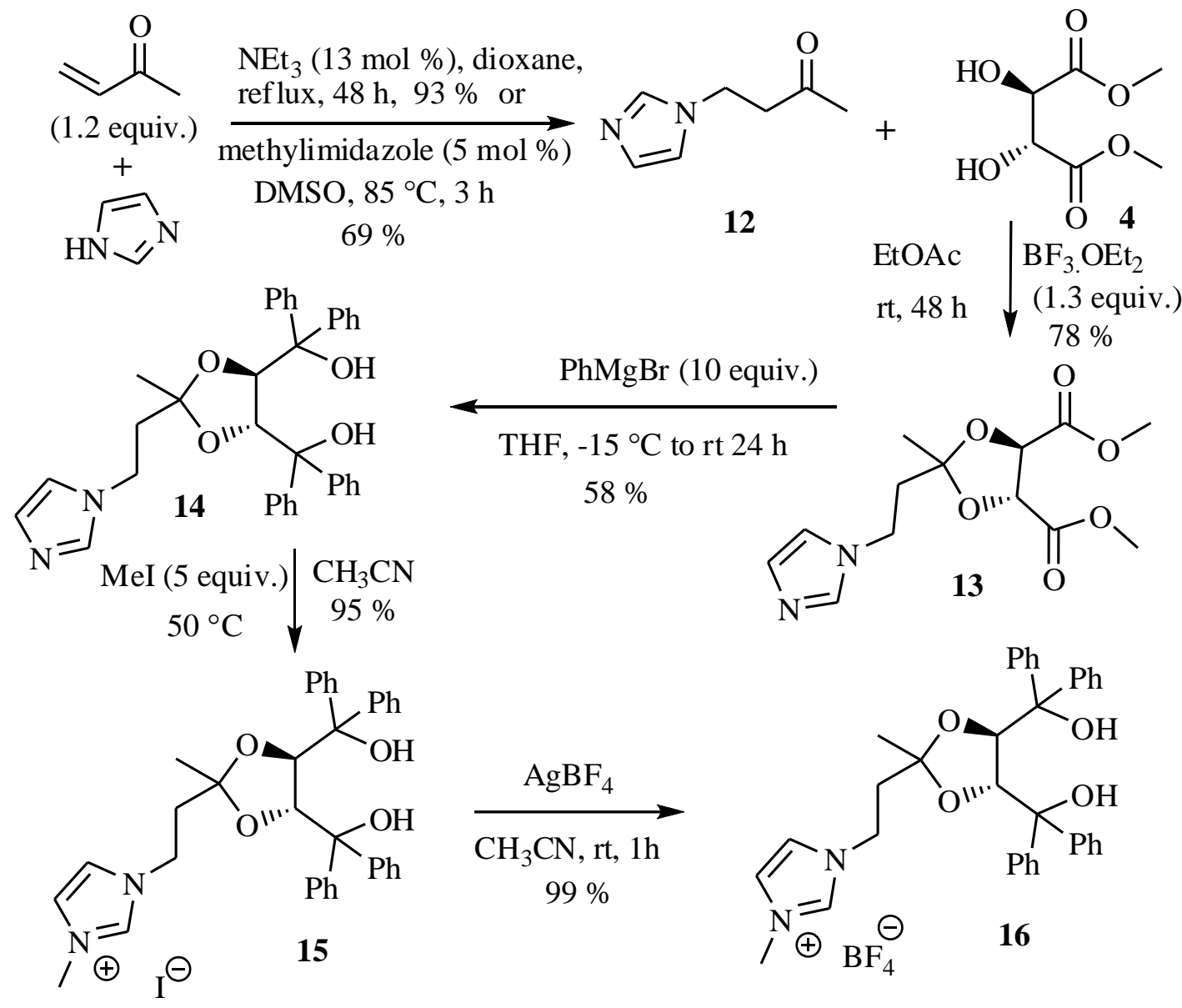

Scheme 2. Synthesis of imidazolium ionic liquid tagged TADDOL catalysts $\mathbf{1 5}$ and $\mathbf{1 6 .}$

In our cases the best yield (58\%) was obtained with the naphthyl TADDOL 11c at room temperature. As shown with the catalyst 11d (entries 8-10, Table 1) lowering the reaction temperature resulted in diminished yields. This effect can also be attributed to the fact that the viscosity of the mixture increased which hampers miscibility of the reaction solution and causes crystallization of the catalyst. In order to alleviate this problem, additional solvents such as toluene, dichloromethane and carbon tetrachloride were used. Non-polar solvents such as toluene often being considered to increase selectivity, ${ }^{31}$ furnished only trace amounts of the product in our case (not shown). This can be ascribed to the low solubility of the IL-tagged catalysts in nonpolar solvents. The more polar solvent dichloromethane and carbon tetrachloride did not improve the yield or enantioselectivity (entries 11,12, Table 1). 
<smiles>C=C(C=C(OC)OC)O[Na]</smiles>

Table 1. Selected examples of HDA reaction between Brassard's diene $\mathbf{1 7}$ and benzaldehyde

\begin{tabular}{ccccc}
\hline Entry & Catalyst & Solvent & $\mathrm{T}^{\circ} \mathrm{C}$ & $\%$ Yield $^{\mathrm{a}}$ \\
\hline 1 & $\mathbf{1 0 a}$ & - & 24 & 49 \\
2 & $\mathbf{1 0 b}$ & - & 24 & 52 \\
3 & $\mathbf{1 1 c}$ & - & 24 & 58 \\
4 & $\mathbf{1 0 d}$ & - & 0 & 32 \\
5 & $\mathbf{1 1 a}$ & - & -24 & 11 \\
6 & $\mathbf{1 1 b}$ & - & 24 & 47 \\
7 & $\mathbf{1 1 c}$ & - & 0 & 36 \\
8 & $\mathbf{1 1 d}$ & - & 24 & 22 \\
9 & $\mathbf{1 1 d}$ & - & -24 & 12 \\
10 & $\mathbf{1 1 d}$ & - & -78 & - \\
11 & $\mathbf{1 1 d}$ & $\mathrm{CH}_{2} \mathrm{Cl}_{2}$ & -24 & 10 \\
12 & $\mathbf{1 1 d}$ & $\mathrm{CCl}_{4}$ & -24 & 14 \\
\hline
\end{tabular}

a isolated yields.

The 1,2,3-triazolium and imidazolium tagged organocatalysts were further tested in HDA reaction of Rawal's diene 19 with benzaldehyde (Table 2). The reactions were carried out with 510 molar equivalent of benzaldehyde in the presence of $20 \mathrm{~mol} \%$ of catalyst. At $0{ }^{\circ} \mathrm{C}$ and in the case of $10 \mathrm{~d}, \mathbf{1 1 b}$ and 13 even at $-24{ }^{\circ} \mathrm{C}$ the intended cycloaddition adducts 20 were obtained in a non-enantioselective fashion in low yields. It turned out that phenyl substituted triazoliumtagged TADDOLs are less effective than 1-naphthyl-substituted TADDOLs (Table 2, compare enties 1, 2 and 3 or entries 3 and 5). Catalysts $\mathbf{1 1 b}$ and $\mathbf{1 5}$ even failed to give a product at $-24{ }^{\circ} \mathrm{C}$ (entries 5 and 12). A more systematic variation of conditions was performed with catalyst 11d. Here, low enantioselectivities (maximum $26 \%$ ee) were observed when the catalyst 11d (20 mol\%) was used in the reactions conducted at -24 and $-78{ }^{\circ} \mathrm{C}$ (entries $7-8$ ). Lowering the catalyst loading to $10 \mathrm{~mol} \%$ resulted in a decrease in yield while the enantioselectivity was not affected (Table 2, entries 7 and 9) Since unlike Brassard's diene Rawal's diene is reactive enough to undergo HDA reaction with benzaldehyde without the aid of any catalysts at room temperature the uncatalyzed cycloaddition is likely to run as background reaction thus giving low enantioselectivities or even racemic products. ${ }^{32-34}$ 


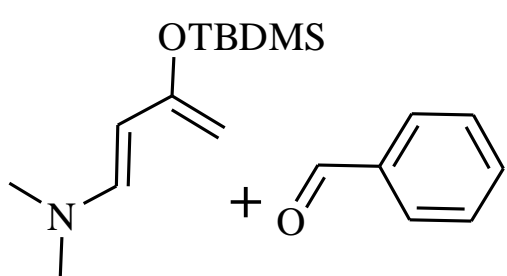

19

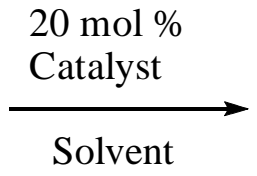

Solvent

Table 2. Applications of 1,2,3-triazolium and imidazolium IL-tagged TADDOL catalysts in the HDA reaction between Rawal's diene 19 and benzaldehyde

\begin{tabular}{llllll}
\hline Entry & Catalyst & Solvent & $\mathrm{T}^{\circ} \mathrm{C}$ & $\%$ Yield $^{\mathrm{a}}$ & $\%$ ee \\
\hline 1 & $\mathbf{1 0 b}$ & - & 0 & 26 & - \\
2 & $\mathbf{1 0 c}$ & - & 0 & 37 & - \\
3 & $\mathbf{1 0 d}$ & - & -24 & 11 & - \\
4 & $\mathbf{1 1 a}$ & - & 0 & 17 & - \\
5 & $\mathbf{1 1 b}$ & & -24 & - & - \\
6 & $\mathbf{1 1 d}$ & - & 0 & 34 & - \\
7 & $\mathbf{1 1 d}$ & - & -24 & 41 & 12 \\
8 & $\mathbf{1 1 d}$ & - & -78 & 32 & 26 \\
9 & $\mathbf{1 1 d}$ & - & -24 & 19 & 12 \\
10 & $\mathbf{1 1 d}$ & $\mathrm{CH}_{2} \mathrm{Cl}_{2}$ & -24 & 16 & - \\
11 & $\mathbf{1 1 d}$ & $\mathrm{CCl}_{4}$ & -24 & 14 & - \\
12 & $\mathbf{1 5}$ & - & -24 & - & - \\
13 & $\mathbf{1 6}$ & - & -24 & 17 & - \\
\hline
\end{tabular}

${ }^{\mathrm{a}}$ isolated yields. ${ }^{\mathrm{b}} 10 \mathrm{~mol} \%$ catalyst.

The fact that 1-naphthyl-substituted TADDOLs perform better than phenyl-substituted ones in terms of enantioselectivity was well documented in the literature for untagged TADDOL catalysts. ${ }^{30}$ This improved efficiency is attributed to the better steric shielding ability of the naphthyl group and the stronger $\pi-\pi$ donor-acceptor interaction ( $\pi$-stacking) between the naphthyl substituents and the aldehyde.

When the IL-tagged TADDOLs were applied to the cycloaddition with Rawal's diene an increase in the viscosity of the reaction medium and crystallization of the catalyst was observed with decreasing temperature as in the case of Brassard's diene (v.s.). To circumvent this obstacle small amounts of toluene were added. However, this did not improve the solubility of the catalyst at lower temperatures. . When $\mathrm{CH}_{2} \mathrm{Cl}_{2}$ (entry 10, Table 2) and $\mathrm{CCl}_{4}$ (entry 11, Table 2) were used as solvents no enantioselectivities were observed and the yields were lower. Attempts to improve the selectivity by increasing the amount of IL-tagged organocatalyst at various temperatures had shown incomplete dissolution of the catalyst in the reaction mixture. Consequently, the investigations were made at a catalyst concentration of $20 \%$ or less. 


\section{Conclusions}

In summary, ionic liquid tagged TADDOLs are reported for the first time. 1,2,3-Triazolium or imidazolium units were used as ionic liquid tags. The former were obtained via Meldal-Sharpless "click"-reaction of propargyloxy-substituted TADDOLs and N-methylation of the resulting 1,2,3-triazoles eventually followed by salt metathesis with silver tetrafluoroborate. The imidazolium moiety was introduced by the Michael-addition of imidazole to methyl vinyl ketone and building up the dioxolane ring with dimethyl tartrate followed by Grignard reaction and Nmethylation. Both synthetic routes are straight forward and allow the preparation of the ILtagged TADDOLs in good yields. The IL-tagged TADDOLs were applied to hetero-Diels Alder reactions between Brassard's diene or Rawals's diene and benzaldehyde. In the former case only racemic cycloaddition products were obtained while in latter case modest enantioselectivities were observed.

\section{Experimental Section}

General. ${ }^{1} \mathrm{H}-\mathrm{NMR}$ and ${ }^{13} \mathrm{C}-\mathrm{NMR}$ solution spectra were recorded at $300 \mathrm{MHz}$ and $75 \mathrm{MHz}$, respectively; with a Bruker $\mathrm{AC} 300$ in $\mathrm{CDCl}_{3}$ as the solvent in $5 \mathrm{~mm}$ NMR tubes with TMS as internal standard. The assignment of ${ }^{13} \mathrm{C}$ signals is based on APT measurements. HRMS was obtained with a Waters UPLC, LCT Premier XE instrument. Determination of enantioselectivities was achieved using chiral columns (Daicel Chemical Industries LTD), mobile phase: $n$-hexane : $i$-propanol (98:20 - 80:20), flow rate: $0.5-1.0 \mathrm{~mL} / \mathrm{min}$. The optical rotations were measured on a Perkin Elmer-241 polarimeter. The measurements were made at a wavelength of $589 \mathrm{~nm}$ using $100 \times 3.5 \mathrm{~mm}$ cuvette.

3-Propargyloxybenzaldehyde (3). A solution of 3-hydroxybenzaldehyde (3.66 g, $30 \mathrm{mmol})$ in DMF $(50 \mathrm{~mL})$ was placed in a two necked flask fitted with an efficient reflux condenser. $\mathrm{Na}_{2} \mathrm{CO}_{3}$ (12.45 g, $90 \mathrm{mmol}$ ) was added. The reaction mixture was heated to $55-60{ }^{\circ} \mathrm{C}$ for half an hour and was cooled to room temperature. Then propargyl bromide $80 \%$ in toluene $(4.05 \mathrm{~mL}, 36$ mmol) was added. The reaction mixture was stirred at the same temperature while its progress was being followed by TLC. After completion (about $4 \mathrm{~h}$ ) the mixture was poured into ice water $(100 \mathrm{~mL})$ with stirring. The resulting solution was extracted with $\mathrm{Et}_{2} \mathrm{O}(100 \mathrm{~mL})$ three times. The combined organic extracts were washed with additional cold water $(100 \mathrm{~mL})$ two times and dried with $\mathrm{MgSO}_{4}$. The organic solvent was removed under reduced pressure to furnish the title product as a yellowish liquid. Yield 92\%. ${ }^{1} \mathrm{H}$ NMR $\left(300 \mathrm{MHz} C D C l_{3}\right): \delta \mathrm{ppm}=10.0(\mathrm{~s}, 1 \mathrm{H})$, 7.68 - 7.37 (m, $3 \mathrm{H}), 7.32-7.16(\mathrm{~m}, 1 \mathrm{H}), 4.74(\mathrm{~d}, J=2.41,2 \mathrm{H}), 2.57$ (t, $J=2.39,2.39 \mathrm{~Hz}, 1$ H). ${ }^{13} \mathrm{C}$ NMR $\left(75 \mathrm{MHz}, C D C l_{3}\right): \delta \mathrm{ppm}=191.8(\mathrm{Ph}-\underline{\mathrm{CH}}=\mathrm{O}), 158.0\left(\underline{\mathrm{C}}_{\mathrm{ph}}-\mathrm{O}\right), 137.7\left(\underline{\mathrm{C}}_{\mathrm{ph}}-\mathrm{CHO}\right)$, 
130.1 - $113.5\left(\underline{\mathrm{CH}}_{\mathrm{ph}}\right), 77.9\left(\mathrm{CH}_{2}-\underline{\mathrm{C}} \equiv \mathrm{CH}\right), 76.2\left(\mathrm{CH}_{2}-\mathrm{C} \equiv \underline{\mathrm{C}} \mathrm{H}\right), 55.9\left(\underline{\mathrm{CH}}_{2}-\mathrm{C} \equiv \mathrm{CH}\right) . \mathrm{HRMS}(\mathrm{ESI})$ : Found: $m / z$ 159.0480. Calculated for $\mathrm{C}_{10} \mathrm{H}_{7} \mathrm{O}_{2}\left(\mathrm{M}-\mathrm{H}^{+}\right)$: 159.0446 .

(4R,5R)-2-(3-(Prop-2-ynyloxy)phenyl)-1,3-dioxolane-4,5-dicarboxylate dimethyl ester (5). A solution of 3-propargyloxybenzaldehyde 3 ( $4.3 \mathrm{~g}, 26.9 \mathrm{mmol})$ in dry benzene $(30 \mathrm{~mL})$ at $0{ }^{\circ} \mathrm{C}$ was kept in a distillation set up under a steady flow of argon. (Caution: benzene is a carcinogenic substance). $p$-TsOH catalyst $(172 \mathrm{mg}, 1 \mathrm{mmol})$ was added followed by trimethyl orthoformate (TMOF) $(4 \mathrm{~mL}, 30 \mathrm{mmol})$. The mixture was stirred at the same temperature for half an hour and $L$-(+)-dimethyl tartrate 4 (5.3 g, $30 \mathrm{mmol})$ was added. Then, it was heated gently to $60{ }^{\circ} \mathrm{C}$, at which point an azeotropic distillate started. N.B.The temperature of the heating oil bath was kept between $75-85{ }^{\circ} \mathrm{C}$ until the benzene/methanol azeotrope boiling at about $57{ }^{\circ} \mathrm{C}$ has ceased to distil off. The heating was continued until the temperature of the distillate increases to $80{ }^{\circ} \mathrm{C}$. After the completion of the reaction was confirmed by TLC the remaining benzene was distilled off under reduced pressure within a well ventilated fume hood. The resulting oil was diluted with $\mathrm{CH}_{2} \mathrm{Cl}_{2}$, neutralized by $\mathrm{Et}_{3} \mathrm{~N}$ and washed with distilled water $(100 \mathrm{~mL})$ and brine $(100 \mathrm{~mL})$ to furnish the title product $(6)$ as a pale yellow oil. Yield $84 \% .{ }^{1} \mathrm{H}$ NMR (300 MHz CDCl $): \delta=7.37-7.28(\mathrm{~m}, 1 \mathrm{H}), 7.23-7.18(\mathrm{~m}, 2 \mathrm{H}), 7.03-6.99(\mathrm{~m}, 1 \mathrm{H})$, $6.12(\mathrm{~m}, 1 \mathrm{H}), 4.98(\mathrm{~d}, J=3.96 \mathrm{~Hz}, 1 \mathrm{H}), 4.70(\mathrm{~d}, J=2.40 \mathrm{~Hz}, 1 \mathrm{H}), 4.66(\mathrm{~m}, 2 \mathrm{H}), 3.86(\mathrm{~s}, 3 \mathrm{H})$, $3.81(\mathrm{~s}, 3 \mathrm{H}), 2.54(\mathrm{t}, J=2.40,2.40 \mathrm{~Hz}, 1 \mathrm{H}) .{ }^{13} \mathrm{C} \mathrm{NMR}\left(75 \mathrm{MHz}, \mathrm{CDCl}_{3}\right): \delta=169.9,169.4$ $\left(\mathrm{O}=\underline{\mathrm{C}}-\mathrm{O}-\mathrm{CH}_{3}\right), 157.6\left(\underline{\mathrm{C}}_{\mathrm{ph}} \mathrm{O}\right), 136.9\left(\underline{\mathrm{C}}_{\mathrm{ph}}-\mathrm{CH}-\mathrm{O}\right), 129.5$ - $113.2\left(\underline{\mathrm{CH}}_{\mathrm{ph}}\right), 106.3\left(\mathrm{C}_{\mathrm{ph}}-\underline{\mathrm{CH}}-\mathrm{O}_{2}\right), 78.4$ $\left(\mathrm{CH}_{2}-\underline{\mathrm{C}} \equiv \mathrm{CH}\right), 77.4\left(\mathrm{CH}_{2}-\mathrm{C} \equiv \underline{\mathrm{CH}}\right), 77.1$ (O- $\left.\underline{\mathrm{CH}}-\mathrm{CH}-\mathrm{O}\right), 76.7(\mathrm{O}-\mathrm{CH}-\underline{\mathrm{CH}}-\mathrm{O}), 55.8\left(\underline{\mathrm{CH}}_{2}-\mathrm{C} \equiv \mathrm{CH}\right)$, $52.9\left(\mathrm{O}=\mathrm{C}-\mathrm{O}-\underline{C H}_{3}\right)$. HRMS (ESI): Found: $m / z$ 319.0819. Calculated for $\mathrm{C}_{16} \mathrm{H}_{15} \mathrm{O}_{7}\left(\mathrm{M}-\mathrm{H}^{+}\right)$: 319.0818. $[\alpha]_{D}^{28}=-19.4\left(c=1, \mathrm{CHCl}_{3}\right)$.

((4R,5R)-(2-(3-(Prop-2-ynyloxy)phenyl)-1,3-dioxolane-4,5-diyl)bis(diphenylmethanol) (7a). Grignard reagent was prepared from magnesium turnings $(2.0 \mathrm{~g}, 83 \mathrm{mmol})$ in dry THF $(80 \mathrm{~mL})$ with a slow addition of bromobenzene $(12.56 \mathrm{~g}, 80 \mathrm{mmol})$ in a three neck round-bottom flask fitted with a efficient condenser which has been oven dried and flushed with argon. The reaction mixture was set to reflux by itself with a controlled addition of the bromobenzene. After the complete consumption of the $\mathrm{Mg}$ tunings refluxing was continued for additional one hour using a water-bath. After this time the mixture was allowed to cool to room temperature and was further cooled to $-15^{\circ} \mathrm{C}$. To it was added 2-(3-(prop-2-ynyloxy)phenyl)-1,3-dioxolane-4,5-dicarboxylate dimethyl ester 6 (3.2 g, $10 \mathrm{mmol})$ in dry THF (30 mL) while keeping the temperature below -10 ${ }^{\circ} \mathrm{C}$. After complete addition of $\mathbf{6}$ the reaction flask was allowed to warm to room temperature and was left to stir overnight. The resulting solution was diluted by $\mathrm{Et}_{2} \mathrm{O}(200 \mathrm{~mL})$ and quenched by concentrated $\mathrm{NH}_{4} \mathrm{Cl} 100 \mathrm{~mL}$ at $0{ }^{\circ} \mathrm{C}$. The ether phase was separated and dried with $\mathrm{MgSO}_{4}$, the solvent was removed under vacuum to give a yellow solid, which was purified by silica gel column chromatography using cyclohexane: ethyl acetate $(7.5: 2.5)$ to furnish pure product as yellowish oil. Yield 63\%. ${ }^{1} \mathrm{H}$ NMR $\left(300 \mathrm{MHz} \mathrm{CDCl}_{3}\right) \delta=7.54-6.88$ (m, $\left.24 \mathrm{H}\right), 5.47(\mathrm{~s}, 1 \mathrm{H})$, $5.30(\mathrm{~s}, 1 \mathrm{H}), 5.27(\mathrm{~s}, 1 \mathrm{H}), 4.64(\mathrm{dd}, J=8.57,2.42 \mathrm{~Hz}, 2 \mathrm{H}), 2.52$ (,t, $J=2.40,2.40 \mathrm{~Hz}, 1 \mathrm{H})$. ${ }^{13} \mathrm{C}$ NMR $\left(75 \mathrm{MHz}, \mathrm{CDCl}_{3}\right) \delta=157.8\left(\underline{\mathrm{C}}_{\mathrm{ph}} \mathrm{O}\right), 166.9\left(\underline{\mathrm{C}}_{\mathrm{ph}} \mathrm{C}-\mathrm{OH}\right), 136.9\left(\underline{\mathrm{C}}_{\mathrm{ph}}-\mathrm{CH}-\mathrm{O}\right), 129.5$ $113.2\left(\underline{\mathrm{CH}}_{\mathrm{ph}}\right), 106.3\left(\mathrm{C}_{\mathrm{ph}}-\underline{\mathrm{C}} \mathrm{H}-\mathrm{O}_{2}\right), 79.9\left(\mathrm{CH}_{2}-\underline{\mathrm{C}} \equiv \mathrm{CH}\right), 79.4\left(\mathrm{CH}_{2}-\mathrm{C} \equiv \underline{\mathrm{C}} \mathrm{H}\right), 78.1$ (O- $\left.\underline{\mathrm{C}}-\mathrm{CH}-\mathrm{O}\right)$, 
77.7 (O-CH-ChH-O), $56.8\left(\underline{\mathrm{CH}}_{2}-\mathrm{C} \equiv \mathrm{CH}\right)$. HRMS (ESI): Found: $\mathrm{m} / \mathrm{z}$ 567.2074. Calculated for $\mathrm{C}_{38} \mathrm{H}_{31} \mathrm{O}_{5}\left(\mathrm{M}-\mathrm{H}^{+}\right): 567.2071$.

((4R,5R)-(2-(3-(Prop-2-ynyloxy)phenyl)-1,3-dioxolane-4,5-diyl)bis(bis(3,5-dimethylphenyl) methanol) (7b). By analogous procedure as 7a from 3,5-dimethyl-bromobenzene (11.1 g, 80 mmol) and 6 (3.2 g, $10 \mathrm{mmol})$. Yield 69\%. $\left.{ }^{1} \mathrm{H} \mathrm{NMR} \mathrm{(300} \mathrm{MHz} \mathrm{CDCl}_{3}\right): \delta=7.42-6.58(\mathrm{~m}, 16$ H), $5.53(\mathrm{~s}, 1 \mathrm{H}), 5.36(\mathrm{~s}, 1 \mathrm{H}), 5.22(\mathrm{~s}, 1 \mathrm{H}), 4.64(\mathrm{dd}, J=8.57,2.42 \mathrm{~Hz}, 2 \mathrm{H}), 2.53$ - 2.19 ( m, $24 \mathrm{H}), 1.96$ - 1.85 ( m, $1 \mathrm{H}) .{ }^{13} \mathrm{C} \mathrm{NMR}\left(75 \mathrm{MHz}, \mathrm{CDCl}_{3}\right): \delta=160.0\left(\underline{\mathrm{C}}_{\mathrm{ph}} \mathrm{O}\right), 155.9-113.9\left(\underline{\mathrm{C}}_{\mathrm{ph}}\right.$ and $\left.\underline{\mathrm{CH}}_{\mathrm{ph}}\right), 104.9\left(\mathrm{C}_{\mathrm{ph}}-\underline{\mathrm{C}} \mathrm{H}-\mathrm{O}_{2}\right), 81.6\left(\mathrm{CH}_{2}-\underline{\mathrm{C}} \equiv \mathrm{CH}\right), 81.0\left(\mathrm{CH}_{2}-\mathrm{C} \equiv \underline{\mathrm{CH}}\right), 79.1(\mathrm{O}-\underline{\mathrm{C}} \mathrm{H}-\mathrm{CH}-\mathrm{O}), 78.6$ (O-CH- $\underline{\mathrm{CH}}-\mathrm{O}), 60.7\left(\underline{\mathrm{CH}}_{2}-\mathrm{C} \equiv \mathrm{CH}\right), 52.9\left(\mathrm{O}=\mathrm{C}-\mathrm{O}-\underline{\mathrm{C}} \mathrm{H}_{3}\right), 21.7$ - $19.5\left(\mathrm{Ph}^{-} \underline{\mathrm{CH}}_{3}\right) . \mathrm{HRMS}(\mathrm{ESI})$ : Found: $m / z$ 679.3419. Calculated for $\mathrm{C}_{46} \mathrm{H}_{47} \mathrm{O}_{5}\left(\mathrm{M}-\mathrm{H}^{+}\right): 679.3423$.

\section{((4R,5R)-2-(3-(Prop-2-ynyloxy)phenyl)-1,3-dioxolane-4,5-diyl)bis(dinaphthalen-1-}

ylmethanol) (7c). By analogous procedure as 7a and 7b from 1-naphthylbromide (10.49 g, 50 $\mathrm{mmol})$ and 6 (3.2 g, $10 \mathrm{mmol})$. Yield $\left.81 \% .{ }^{1} \mathrm{H} \mathrm{NMR} \mathrm{(300} \mathrm{MHz} \mathrm{CDCl}_{3}\right): \delta=8.67-6.89(\mathrm{~m}, 32$ $\mathrm{H}), 5.54(\mathrm{~s}, 1 \mathrm{H}), 5.35(\mathrm{~s}, 1 \mathrm{H}), 5.22(\mathrm{~s}, 1 \mathrm{H}), 4.63(\mathrm{dd}, J=8.61,2.40 \mathrm{~Hz}, 2 \mathrm{H}), 1.94$ - $1.86(\mathrm{~m}, 1$ $\mathrm{H}) .{ }^{13} \mathrm{C}$ NMR $\left(75 \mathrm{MHz}, \mathrm{CDCl}_{3}\right) \delta=159.9$ - $118.0\left(\underline{\mathrm{C}}_{\mathrm{ph}}\right.$ and $\left.\underline{\mathrm{C}} \mathrm{H}_{\mathrm{ph}}\right), 104.9\left(\mathrm{C}_{\mathrm{ph}}-\underline{\mathrm{C}} \mathrm{H}-\mathrm{O}_{2}\right), 83.2(\mathrm{O}-$ $\underline{\mathrm{CH}}-\mathrm{CH}-\mathrm{O}), 82.9$ (O-CH- $\underline{\mathrm{CH}}-\mathrm{O}), 80.0$ (Ph- $\underline{\mathrm{C}}-\mathrm{OH}), 79.8(\mathrm{Ph}-\underline{\mathrm{C}}-\mathrm{OH}), 79.1 \quad\left(\mathrm{CH}_{2}-\underline{\mathrm{C}} \equiv \mathrm{CH}\right), 78.6$ $\left(\mathrm{CH}_{2}-\mathrm{C} \equiv \underline{\mathrm{CH}}\right), 56.9\left(\underline{\mathrm{CH}}_{2}-\mathrm{C} \equiv \mathrm{CH}\right)$. HRMS (ESI): Found: $m / z$ 767.2784. Calculated for $\mathrm{C}_{54} \mathrm{H}_{39} \mathrm{O}_{5}$ $\left(\mathrm{M}-\mathrm{H}^{+}\right): 767.2797$.

((4R,5R)-(2-(3-((1-Butyl-1H-1,2,3-triazol-4-yl)methoxy)phenyl)-1,3-dioxolane-4,5-diyl)bis(diphenylmethanol) (9a). (4R,5R)-(2-(3-(Prop-2-ynyloxy)phenyl)-1,3-dioxolane-4,5-diyl) bis(diphenylmethanol) 7a (1 g, 1,76 mmol) was dissolved in DMF : MeOH, 1:1 (20 mL). Butyl azide $(174 \mathrm{mg}, 1.76 \mathrm{mmol})$ and $\mathrm{CuSO}_{4}(71 \mathrm{mg}, 0.44 \mathrm{mmol})$ was added followed by sodium ascorbate $(70 \mathrm{mg}, 0.35 \mathrm{mmol})$ with vigorous stirring. The mixture was left to stir for three days. The resulting solution was diluted with $\mathrm{CH}_{2} \mathrm{Cl}_{2}(200 \mathrm{~mL})$ and was washed several times with ice cold water. The organic phase was separated and dried with $\mathrm{MgSO}_{4}$ and the solvent was removed under vacuum to give a yellow solid, which was purified by column chromatography using cyclohexane: ethyl acetate $(7.5: 2.5)$ to furnish the title product 9a. Yield $82 \%$. ${ }^{1} \mathrm{H}$ NMR $(300$ $\left.\mathrm{MHz} \mathrm{CDCl}_{3}\right): \delta=7.73-7.13(\mathrm{~m}, 22 \mathrm{H}), 7.03-6.76(\mathrm{~m}, 3 \mathrm{H}), 5.37(\mathrm{~d}, J=4.97 \mathrm{~Hz}, 1 \mathrm{H}), 5.24$ $(\mathrm{s}, 1 \mathrm{H}), 5.20(\mathrm{~d}, J=4.96 \mathrm{~Hz}, 1 \mathrm{H}), 5.09(\mathrm{~d}, J=2.69 \mathrm{~Hz}, 2 \mathrm{H}), 4.33(\mathrm{t}, J=7.23,7.23 \mathrm{~Hz}, 2 \mathrm{H})$, $1.96-1.82(\mathrm{~m}, 2 \mathrm{H}), 1.38(\mathrm{dd}, J=14.77,7.33 \mathrm{~Hz}, 2 \mathrm{H}), 0.99(\mathrm{t}, J=7.38,7.38 \mathrm{~Hz}, 3 \mathrm{H}) .{ }^{13} \mathrm{C}$ NMR $\left(75 \mathrm{MHz}, \mathrm{CDCl}_{3}\right) \delta=158.1\left(\mathrm{O}-\underline{\mathrm{C}}_{\mathrm{ph}}\right), 146.0-113.0\left(\mathrm{~N}-\underline{\mathrm{C}} \mathrm{H}=\mathrm{C}, \underline{\mathrm{CH}}_{\mathrm{ph}}\right.$ and $\left.\underline{\mathrm{C}}_{\mathrm{ph}}\right), 104.6(\mathrm{C}-$ $\left.\mathrm{CH}_{2}-\underline{\mathrm{CH}}-\mathrm{O}_{2}\right), 81.6$ (O- $\left.\underline{\mathrm{CH}}-\mathrm{CH}-\mathrm{O}\right), 80.9$ (O-CH- $\left.\underline{\mathrm{CH}}-\mathrm{O}\right), 78.6\left((\mathrm{Ph})_{2}-\underline{\mathrm{C}}-\mathrm{OH}\right), 78.5\left((\mathrm{Ph})_{2}-\underline{\mathrm{C}}-\mathrm{OH}\right)$,

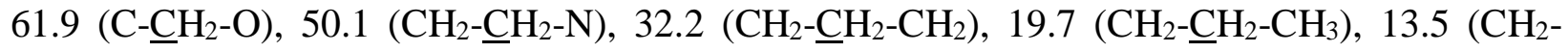
$\mathrm{CH}_{2}-\mathrm{CH}_{3}$ ). HRMS (ESI): Found: $m / z$ 666.2969. Calculated for $\mathrm{C}_{42} \mathrm{H}_{40} \mathrm{~N}_{3} \mathrm{O}_{5}\left(\mathrm{M}-\mathrm{H}^{+}\right)$: 666.2968. $[\alpha]_{D}^{29}=+38.8^{\circ}\left(c=1, \mathrm{CCl}_{3}\right)$.

\section{((4R,5R)-(2-(3-((1-Butyl-1H-1,2,3-triazol-4-yl)methoxy)phenyl)-1,3-dioxolane-4,5-}

diyl)bis(di(3,5-dimethylphenyl)methanol) (9b). By analogous procedure as 9a from (4R,5R)(2-(3-(prop-2-ynyloxy)phenyl)-1,3-dioxolane-4,5-diyl)bis(bis(3,5-dimethylphenyl)methanol) 7b, (4.5 g, $6.6 \mathrm{mmol})$ and butyl azide $(680 \mathrm{mg}, 6.9 \mathrm{mmol})$. Yield 94\%. M.p. $81-84{ }^{\circ} \mathrm{C} .{ }^{1} \mathrm{H}$ NMR $\left(300 \mathrm{MHz} \mathrm{CDCl}_{3}\right): \delta=7.33-6.71(\mathrm{~m}, 17 \mathrm{H}), 5.45(\mathrm{~d}, J=3.91 \mathrm{~Hz}, 1 \mathrm{H}), 5.38(\mathrm{~s}, 1 \mathrm{H}), 5.24(\mathrm{~d}, J$ 
$=4.58 \mathrm{~Hz}, 1 \mathrm{H}), 5.23(\mathrm{~m}, 2 \mathrm{H}), 4.34(\mathrm{t}, J=7.23,7.23 \mathrm{~Hz}, 2 \mathrm{H}), 2.46-2.25(\mathrm{~m}, 24 \mathrm{H}), 1.96-1.82$ $(\mathrm{m}, 2 \mathrm{H}), 1.56(\mathrm{dd}, J=14.77,7.33 \mathrm{~Hz}, 2 \mathrm{H}), 0.99(\mathrm{t}, J=7.38,7.38 \mathrm{~Hz}, 3 \mathrm{H}) .{ }^{13} \mathrm{C}$ NMR $(75$ $\left.\mathrm{MHz}, \mathrm{CDCl}_{3}\right): \delta=159.8\left(\mathrm{O}-\underline{\mathrm{C}}_{\mathrm{ph}}\right), 155.7-113.7\left(\mathrm{~N}-\underline{\mathrm{CH}}=\mathrm{C}, \mathrm{CH}=\underline{\mathrm{C}}-\mathrm{CH}_{2}, \underline{\mathrm{CH}}_{\mathrm{ph}}\right.$ and $\left.\underline{\mathrm{C}}_{\mathrm{ph}}\right), 104.6(\mathrm{C}-$ $\left.\mathrm{CH}_{2}-\underline{\mathrm{CH}}-\mathrm{O}\right), 81.4$ (O- $\left.\underline{\mathrm{CH}}-\mathrm{CH}-\mathrm{O}\right), 80.7$ (O-CH- $\left.\underline{\mathrm{CH}}-\mathrm{O}\right), 78.9$ ((Ph) $\left.)_{2}-\underline{\mathrm{C}}-\mathrm{OH}\right), 78.3\left((\mathrm{Ph})_{2}-\underline{\mathrm{C}}-\mathrm{OH}\right)$, $60.5\left(\mathrm{C}-\underline{\mathrm{CH}}_{2}-\mathrm{O}\right), 52.6\left(\mathrm{CH}_{2}-\underline{\mathrm{CH}}_{2}-\mathrm{N}\right), 26.8\left(\mathrm{CH}_{2}-\underline{\mathrm{CH}}_{2}-\mathrm{CH}_{2}\right), 21.5-20.8\left(\mathrm{Ph}_{-} \mathrm{CH}_{3}\right), 19.3\left(\mathrm{CH}_{2}-\right.$ $\left.\underline{\mathrm{CH}}_{2}-\mathrm{CH}_{3}\right)$, $14.0 \quad\left(\mathrm{CH}_{2}-\mathrm{CH}_{2}-\underline{\mathrm{CH}}_{3}\right)$. HRMS (ESI): Found: $\mathrm{m} / z$ 778.4222. Calculated for $\mathrm{C}_{50} \mathrm{H}_{56} \mathrm{~N}_{3} \mathrm{O}_{5}\left(\mathrm{M}-\mathrm{H}^{+}\right): 778.4220$.

((4R,5R)-2-(3-((1-Butyl-1H-1,2,3-triazol-4-yl)methoxy)phenyl)-1,3-dioxolane-4,5-

diyl)bis(dinaphthalen-1-ylmethanol) (9c). By analogous procedure as 9a from $((4 R, 5 R)-2-(3-$ (prop-2-ynyloxy)phenyl)-1,3-dioxolane-4,5-diyl)bis(dinaphthalen-1-ylmethanol) 7c (1.53 g, 2 mmol) and butyl azide (200 mg, $2 \mathrm{mmol}$ ) was obtained the title product as colour less oil. Yield 92\%. ${ }^{1} \mathrm{H}$ NMR $\left(300 \mathrm{MHz} \mathrm{CDCl}_{3}\right): \delta \mathrm{ppm}=8.73-7.27(\mathrm{~m}, 33 \mathrm{H}), 5.57(\mathrm{~s}, 1 \mathrm{H}), 5.34(\mathrm{~d}, J=4.01$ $\mathrm{Hz}, 1 \mathrm{H}), 5.30(\mathrm{~d}, J=4.24 \mathrm{~Hz}, 1 \mathrm{H}), 5.14(\mathrm{~d}, J=4.48 \mathrm{~Hz}, 2 \mathrm{H}), 4.35$ (t, $J=7.29,7.29 \mathrm{~Hz}, 2 \mathrm{H})$, $1.93-1.87(\mathrm{~m}, 2 \mathrm{H}), 1.44-1.28(\mathrm{~m}, 2 \mathrm{H}), 0.95(\mathrm{t}, J=7.33,7.33 \mathrm{~Hz}, 3 \mathrm{H}) .{ }^{13} \mathrm{C} \mathrm{NMR}(75 \mathrm{MHz}$, $\left.\mathrm{CDCl}_{3}\right): \delta \mathrm{ppm}=156.5\left(\mathrm{O}-\underline{\mathrm{C}}_{\mathrm{ph}}\right), 148.3-114.1\left(\mathrm{~N}-\underline{\mathrm{CH}}=\mathrm{C}, \mathrm{CH}=\underline{\mathrm{C}}-\mathrm{CH}_{2}, \underline{\mathrm{CH}}_{\mathrm{ph}}\right.$ and $\left.\underline{\mathrm{C}}_{\mathrm{ph}}\right), 106.2(\mathrm{O}-$ $\underline{\mathrm{CH}}-\mathrm{O}), 82.4$ (O- $\underline{\mathrm{CH}}-\mathrm{CH}-\mathrm{O}), 81.8(\mathrm{O}-\mathrm{CH}-\underline{\mathrm{C}} \mathrm{H}-\mathrm{O}), 80.1\left((\mathrm{Ph})_{2}-\underline{\mathrm{C}}-\mathrm{OH}\right), 79.3\left((\mathrm{Ph})_{2}-\underline{\mathrm{C}}-\mathrm{OH}\right), 62.5(\mathrm{C}-$

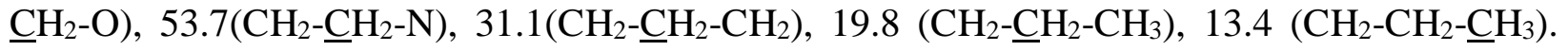
HRMS (ESI): Found: $m / z$ 868.3762. Calculated for $\mathrm{C}_{58} \mathrm{H}_{50} \mathrm{~N}_{3} \mathrm{O}_{5}\left(\mathrm{M}+\mathrm{H}^{+}\right): 868.3760$.

((4R,5R)-2-(3-((1-Benzyl-1H-1,2,3-triazol-4-yl)methoxy)phenyl)-1,3-dioxolane-4,5diyl)bis(dinaphthalen-1-ylmethanol) (9d). By analogous procedure as 9aform ((4R,5R)-2-(3(prop-2-ynyloxy)phenyl)-1,3-dioxolane-4,5-diyl)bis(dinaphthalen-1-ylmethanol) 7c (1.53 g, 2 $\mathrm{mmol}$ ) and benzyl azide (266 mg, $2 \mathrm{mmol}$ ) was obtained the title product as colour less oil. Yield 98\%. ${ }^{1} \mathrm{H}$ NMR (300 $\left.\mathrm{MHz} \mathrm{CDCl}_{3}\right): \delta \mathrm{ppm}=8.69-7.07(\mathrm{~m}, 38 \mathrm{H}), 5.56(\mathrm{~s}, 1 \mathrm{H}), 5.43(\mathrm{~s}, 2 \mathrm{H})$, $5.37(\mathrm{~d}, J=4.01 \mathrm{~Hz}, 1 \mathrm{H}), 5.31(\mathrm{~d}, J=4.24 \mathrm{~Hz}, 1 \mathrm{H}), 5.14(\mathrm{~d}, J=4.48 \mathrm{~Hz}, 2 \mathrm{H}) .{ }^{13} \mathrm{C}$ NMR $(75$ $\left.\mathrm{MHz}, \mathrm{CDCl}_{3}\right): \delta=156.5\left(\mathrm{O}-\underline{\mathrm{C}}_{\mathrm{ph}}\right), 148.5-111.3\left(\mathrm{~N}-\underline{\mathrm{C}} \mathrm{H}=\mathrm{C}, \mathrm{CH}=\underline{\mathrm{C}}-\mathrm{CH}_{2}, \underline{\mathrm{CH}}_{\mathrm{ph}}\right.$ and $\left.\underline{\mathrm{C}}_{\mathrm{ph}}\right), 106.5(\mathrm{O}-$ $\underline{\mathrm{CH}}-\mathrm{O}), 81.5$ (O- $\underline{\mathrm{CH}}-\mathrm{CH}-\mathrm{O}), 81.2$ (O-CH-ㄷH-O), 79.5 ((Ph) $\left.)_{2}-\underline{\mathrm{C}}-\mathrm{OH}\right), 78.7\left((\mathrm{Ph})_{2}-\underline{\mathrm{C}}-\mathrm{OH}\right), 61.8$ $\left(\mathrm{C}-\underline{\mathrm{CH}}_{2}-\mathrm{O}\right), 53.6\left(\mathrm{Ph}-\underline{\mathrm{CH}}_{2}-\mathrm{N}\right)$. HRMS (ESI): Found: $\mathrm{m} / z$ 902.3585. Calculated for $\mathrm{C}_{61} \mathrm{H}_{48} \mathrm{~N}_{3} \mathrm{O}_{5}$ $\left(\mathrm{M}+\mathrm{H}^{+}\right): 902.3594$.

((4R,5R)-4-((3-(4,5-Bis(hydroxydiphenylmethyl)-1,3-dioxolan-2-yl)phenoxy)methyl)-1butyl-3-methyl-1H-1,2,3-triazol-3-ium iodide (10a). ((4R,5R)-(2-(3-((1-butyl-1H-1,2,3-triazol4-yl)methoxy)phenyl)-1,3-dioxolane-4,5-diyl)bis(diphenylmethanol) 9a, (1 g, 1,5 mmol) was dissolved in $\mathrm{CH}_{3} \mathrm{CN}$ ( $2 \mathrm{~mL}$ ) in a two neck sealed flask fitted with efficient reflux condenser and a balloon, to it was injected methyl iodide $(0.94 \mathrm{~mL}, 15 \mathrm{mmol})$. The reaction mixture was heated at $55{ }^{\circ} \mathrm{C}$ overnight. Volatiles were removed from the resulting liquid to furnish yellow solid product. Yield 98\%. M.p. $115-117{ }^{\circ} \mathrm{C} .{ }^{1} \mathrm{H} \mathrm{NMR}\left(300 \mathrm{MHz} \mathrm{CDCl}_{3}\right): \delta \mathrm{ppm}=7.53-7.05(\mathrm{~m}, 22$ H), $7.01-6.89(\mathrm{~m}, 2 \mathrm{H}), 6.82(\mathrm{~d}, J=7.69 \mathrm{~Hz}, 1 \mathrm{H}), 5.40(\mathrm{~d}, J=4.97 \mathrm{~Hz}, 1 \mathrm{H}), 5.32(\mathrm{~d}, J=4.57$ $\mathrm{Hz}, 1 \mathrm{H}), 5.19$ (d, $J=4.72 \mathrm{~Hz}, 1 \mathrm{H}), 5.09$ (d, $J=2.69 \mathrm{~Hz}, 2 \mathrm{H}), 4.46$ (t, $J=7.32,7.32 \mathrm{~Hz}, 2 \mathrm{H})$, 4.20 (s, N-CH $3,3 \mathrm{H}), 1.93-1.78$ (m, $\left.\mathrm{CH}_{2}-\underline{\mathrm{C}}_{2}-\mathrm{CH}_{2}, 2 \mathrm{H}\right), 1.37-1.21$ (m, $\left.\mathrm{CH}_{2}-\mathrm{C}_{2}-\mathrm{CH}_{3}, 2 \mathrm{H}\right)$, $0.89(\mathrm{t}, J=7.34,7.34 \mathrm{~Hz}, 3 \mathrm{H}) .{ }^{13} \mathrm{C} \mathrm{NMR}\left(75 \mathrm{MHz}, \mathrm{CDCl}_{3}\right): \delta \mathrm{ppm}=156.5\left(\mathrm{O}-\underline{\mathrm{C}}_{\mathrm{ph}}\right), 145.4-$ 
$113.8\left(\mathrm{~N}-\underline{\mathrm{C}} \mathrm{H}=\mathrm{C}, \underline{\mathrm{C}} \mathrm{H}_{\mathrm{ph}}, \underline{\mathrm{C}}_{\mathrm{ph}}\right.$ and $\left.\mathrm{CH}=\underline{\mathrm{C}}-\mathrm{CH}_{2}\right), 104.5\left(\mathrm{Ph}-\underline{\mathrm{CH}}-\mathrm{O}_{2}\right), 81.5(\mathrm{O}-\underline{\mathrm{C}} \mathrm{H}-\mathrm{CH}-\mathrm{O}), 80.9(\mathrm{O}-$ $\mathrm{CH}-\underline{\mathrm{C}} \mathrm{H}-\mathrm{O}), 79.1\left((\mathrm{Ph})_{2}-\underline{\mathrm{C}}-\mathrm{OH}\right), 78.7\left((\mathrm{Ph})_{2}-\underline{\mathrm{C}}-\mathrm{OH}\right), 58.7\left(\mathrm{C}-\underline{\mathrm{CH}}_{2}-\mathrm{O}\right), 54.0\left(\mathrm{CH}_{2}-\underline{\mathrm{CH}}_{2}-\mathrm{N}\right), 39.7(\mathrm{~N}-$ $\left.\underline{\mathrm{CH}}_{3}\right), 31.1\left(\mathrm{CH}_{2}-\mathrm{CH}_{2}-\mathrm{CH}_{2}\right), 19.3\left(\mathrm{CH}_{2}-\underline{\mathrm{CH}}_{2}-\mathrm{CH}_{3}\right), 13.4\left(\mathrm{CH}_{2}-\mathrm{CH}_{2}-\underline{C H}_{3}\right)$. HRMS (ESI), Found: $m / z$ 682.3276. Calculated for $\mathrm{C}_{43} \mathrm{H}_{44} \mathrm{~N}_{3} \mathrm{O}_{5}\left(\mathrm{M}^{+}\right): 682.3275$. $[\alpha]_{D}^{28}=+48.7^{\circ}\left(c=1, \mathrm{CCl}_{3}\right)$.

((4R,5R)-4-((3-(4,5-Bis(bis(3,5-dimethylphenyl)(hydroxy)methyl)-1,3-dioxolan-2yl)phenoxy)methyl)-1-butyl-3-methyl-1H-1,2,3-triazol-3-ium iodide (10b). By analogues procedure as 10a from $((4 R, 5 R)-(2-(3-((1-b u t y l-1 H-1,2,3-t r i a z o l-4-y l) m e t h o x y) p h e n y l)-1,3-$ dioxolane-4,5-diyl)bis(bis(3,5-dimethylphenyl)methanol) 9b (1.3 g, 1,66 mmol) and methyl iodide $(1.0 \mathrm{~mL}, 16 \mathrm{mmol})$. Yield 99\%. M.p. $130-135{ }^{\circ} \mathrm{C} .{ }^{1} \mathrm{H} \mathrm{NMR}\left(300 \mathrm{MHz} \mathrm{CDCl}_{3}\right) \delta \mathrm{ppm}=$ $7.43-6.67(\mathrm{~m}, 17 \mathrm{H}), 5.71(\mathrm{~s}, 1 \mathrm{H}), 5.34(\mathrm{~d}, J=4.01 \mathrm{~Hz}, 1 \mathrm{H}), 5.20(\mathrm{~d}, J=4.24 \mathrm{~Hz}, 1 \mathrm{H}), 5.14$ $(\mathrm{d}, J=4.48 \mathrm{~Hz}, 2 \mathrm{H}), 4.55(\mathrm{t}, J=7.29,7.29 \mathrm{~Hz}, 2 \mathrm{H}), 4.22(\mathrm{~s}, 3 \mathrm{H}), 2.36-2.15(\mathrm{~m}, 24 \mathrm{H}), 1.90$ $1.73(\mathrm{~m}, 2 \mathrm{H}), 1.44-1.28(\mathrm{~m}, 2 \mathrm{H}), 0.95(\mathrm{t}, J=7.33,7.33 \mathrm{~Hz}, 3 \mathrm{H}) .{ }^{13} \mathrm{C} \mathrm{NMR}\left(75 \mathrm{MHz}, \mathrm{CDCl}_{3}\right)$ Sppm $156.5\left(\mathrm{O}-\underline{\mathrm{C}}_{\mathrm{ph}}\right), 145.5-113.4\left(\mathrm{~N}-\underline{\mathrm{CH}}=\mathrm{C}, \mathrm{CH}=\underline{\mathrm{C}}-\mathrm{CH}_{2}, \underline{\mathrm{CH}}_{\mathrm{ph}}\right.$ and $\left.\underline{\mathrm{C}}_{\mathrm{ph}}\right), 104.6$ (O-CH-O), 81.5 (O- $\underline{\mathrm{CH}}-\mathrm{CH}-\mathrm{O}), 81.2$ (O-CH-ㄷH-O), $79.5\left((\mathrm{Ph})_{2}-\underline{\mathrm{C}}-\mathrm{OH}\right), 78.7\left((\mathrm{Ph})_{2}-\underline{\mathrm{C}}-\mathrm{OH}\right), 61.8\left(\mathrm{C}-\underline{\mathrm{C}} \mathrm{H}_{2}-\mathrm{O}\right)$, $53.6\left(\mathrm{CH}_{2}-\underline{C H}_{2}-\mathrm{N}\right), 39.7\left(\mathrm{~N}-\underline{\mathrm{C}} \mathrm{H}_{3}\right), 31.2\left(\mathrm{CH}_{2}-\underline{C H}_{2}-\mathrm{CH}_{2}\right), 21.6\left(8 \mathrm{C}, \mathrm{Ph}-\underline{\mathrm{C}} \mathrm{H}_{3}\right), 19.4\left(\mathrm{CH}_{2}-\underline{C H}_{2}-\right.$ $\left.\mathrm{CH}_{3}\right), 13.5\left(\mathrm{CH}_{2}-\mathrm{CH}_{2}-\underline{C H}_{3}\right)$. HRMS (ESI): Found: $m / z$ 794.4527. Calculated for $\mathrm{C}_{51} \mathrm{H}_{60} \mathrm{~N}_{3} \mathrm{O}_{5}$ $\left(\mathrm{M}^{+}\right): 794.4527,[\alpha]_{D}^{28}=+60.5^{\circ}\left(c=1, \mathrm{CHCl}_{3}\right)$.

(4R,5R)-4-((3-(4,5-Bis(hydroxydinaphthalen-1-ylmethyl)-1,3-dioxolan-2-yl)phenoxy)methyl)-1-butyl-3-methyl-1H-1,2,3-triazol-3-ium iodide (10c). By analogous procedure as 10a from $((4 R, 5 R)-2-(3-((1-b u t y l-1 H-1,2,3-t r i a z o l-4-y l) m e t h o x y) p h e n y l)-1,3-d i o x o l a n e-4,5-$ diyl)bis(dinaphthalen-1-ylmethanol) 9d (1.3 g, 1,5 mmol) and methyl iodide (1.0 mL, $16 \mathrm{mmol})$. Yield 94\%. M.p. $177-181{ }^{\circ} \mathrm{C}$. ${ }^{1} \mathrm{H}$ NMR $\left(300 \mathrm{MHz} C D C l_{3}\right) \delta \mathrm{ppm}=8.95-6.49(\mathrm{~m}, 33 \mathrm{H}), 6.05$ $(\mathrm{m}, 1 \mathrm{H}), 5.95(\mathrm{~m}, 1 \mathrm{H}), 5.73(\mathrm{~s}, 1 \mathrm{H}), 4.93(\mathrm{~s}, 2 \mathrm{H}), 4.42(\mathrm{~s}, 3 \mathrm{H}), 4.32(\mathrm{t}, J=7.05,7.05 \mathrm{~Hz}, 2 \mathrm{H})$, $1.87-1.73(\mathrm{~m}, 2 \mathrm{H}), 1.30(\mathrm{dd}, J=14.76,7.32 \mathrm{~Hz}, 2 \mathrm{H}), 0.88(\mathrm{t}, J=7.36,7.36 \mathrm{~Hz}, 3 \mathrm{H}) .{ }^{13} \mathrm{C}$ NMR $\left(75 \mathrm{MHz}, \mathrm{CDCl}_{3}\right): \delta \mathrm{ppm}=158.4\left(\mathrm{O}-\underline{\mathrm{CH}}_{\mathrm{ph}}\right), 143.2-116.2\left(\mathrm{~N}-\underline{\mathrm{CH}}=\mathrm{C}, \mathrm{CH}=\underline{\mathrm{C}}-\mathrm{CH}_{2}, \mathrm{C}_{\mathrm{ph}}\right.$

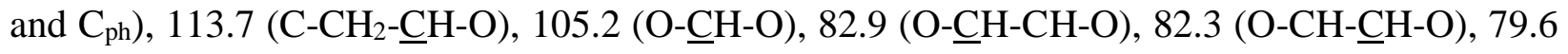
$\left(\mathrm{C}_{\mathrm{ph}}-\mathrm{C}-\mathrm{OH}\right), 62.1\left(\mathrm{C}-\underline{\mathrm{CH}_{2}}-\mathrm{O}\right), 49.7\left(\mathrm{CH}_{2}-\underline{\mathrm{CH}}_{2}-\mathrm{N}\right), 37.8\left(\mathrm{~N}-\mathrm{CH}_{3}\right), 32.0\left(\mathrm{CH}_{2}-\underline{\mathrm{CH}}_{2}-\mathrm{CH}_{2}\right), 19.5$ $\left(\mathrm{CH}_{2}-\mathrm{CH}_{2}-\mathrm{CH}_{3}\right)$, $13.5\left(\mathrm{CH}_{2}-\mathrm{CH}_{2}-\underline{\mathrm{CH}}_{3}\right)$. HRMS (ESI): Found: $m / z$ 882.3898. Calculated for $\mathrm{C}_{59} \mathrm{H}_{52} \mathrm{~N}_{3} \mathrm{O}_{5}\left(\mathrm{M}^{+}\right): 882.3901 .[\alpha]_{D}^{28}=+98.7\left(c=1, \mathrm{CHCl}_{3}\right)$.

(4R,5R)-1-benzyl-4-((3-(4,5-bis(hy droxydinaphthalen-1-ylmethyl)-1,3-dioxolan-2-

yl)phenoxy)methyl)-3-methyl-1H-1,2,3-triazol-3-ium iodide (10d). By analogous procedure as 10a from (( $4 R, 5 R)-2-(3-((1-$ benzyl-1H-1,2,3-triazol-4-yl)methoxy)phenyl)-1,3-dioxolane4,5-diyl)bis(dinaphthalen-1-ylmethanol) 9c (901 mg, $1 \mathrm{mmol})$ and methyl iodide (0.62 mL, 10 mmol) was obtained a yellow solid product. Yield 98\%. ${ }^{1} \mathrm{H} \mathrm{NMR}\left(300 \mathrm{MHz} \mathrm{CDCl}_{3}\right): \delta \mathrm{ppm}=$ 8.74 - 7.17 (m, $38 \mathrm{H}), 5.59$ (s, $1 \mathrm{H}), 5.54(\mathrm{~m}, 2 \mathrm{H}), 5.36$ (d, $J=4.01 \mathrm{~Hz}, 1 \mathrm{H}), 5.33$ (d, $J=4.24$ $\mathrm{Hz}, 1 \mathrm{H}), 5.14(\mathrm{~d}, J=4.48 \mathrm{~Hz}, 2 \mathrm{H}), 3.96(\mathrm{~s}, 3 \mathrm{H}) .{ }^{13} \mathrm{C} \mathrm{NMR}\left(75 \mathrm{MHz}, \mathrm{CDCl}_{3}\right): \delta \mathrm{ppm}=156.5$ $\left(\mathrm{O}-\underline{\mathrm{C}}_{\mathrm{ph}}\right), 148.5-111.3\left(\mathrm{~N}-\underline{\mathrm{C}} \mathrm{H}=\mathrm{C}, \mathrm{CH}=\underline{\mathrm{C}}-\mathrm{CH}_{2}, \underline{\mathrm{CH}}_{\mathrm{ph}}\right.$ and $\left.\underline{\mathrm{C}}_{\mathrm{ph}}\right), 106.5(\mathrm{O}-\underline{\mathrm{C}} \mathrm{H}-\mathrm{O}), 81.5(\mathrm{O}-\underline{\mathrm{C}} \mathrm{H}-\mathrm{CH}-$ $\mathrm{O}), 81.2$ (O-CH-ㄷH-O), $79.5\left((\mathrm{Ph})_{2}-\underline{\mathrm{C}}-\mathrm{OH}\right), 78.7\left((\mathrm{Ph})_{2}-\underline{\mathrm{C}}-\mathrm{OH}\right), 61.8\left(\mathrm{C}-\underline{\mathrm{CH}}{ }_{2}-\mathrm{O}\right), 53.6\left(\mathrm{Ph}_{-} \underline{\mathrm{CH}_{2}-}\right.$ $\mathrm{N}), 37.6\left(\mathrm{~N}_{-} \underline{\mathrm{H}}_{3}\right)$. HRMS (ESI): Found: $\mathrm{m} / \mathrm{z}$ 916.3742. Calculated for $\mathrm{C}_{62} \mathrm{H}_{50} \mathrm{~N}_{3} \mathrm{O}_{5}\left(\mathrm{M}^{+}\right)$: 916.3745 . 
(4R,5R)-4-((3-(4,5-bis(hydroxydiphenylmethyl)-1,3-dioxolan-2-yl)phenoxy)methyl)-1-butyl3-methyl-1H-1,2,3-triazol-3-ium tetrafluoroborate (11a). ((4R,5R)-4-((3-)(4,5bis(hydroxydiphenylmethyl)-1,3-dioxolan-2-yl)phenoxy)methyl)-1-butyl-3-methyl-1H-1,2,3triazol-3-ium iodide 10a (809 mg, $1 \mathrm{mmol}$ ) was dissolved in $\mathrm{CH}_{3} \mathrm{CN}$ (2 mL). To it was added $\mathrm{AgBF}_{4}(195 \mathrm{mg}, 1.0 \mathrm{mmol})$. The mixture was stirred at room temperature for 1 hour. The AgI precipitate was filtered and volatiles were removed from the resulting liquid to furnish as a yellowish viscous liquid product. Yield 98\%. ${ }^{1} \mathrm{H} \mathrm{NMR}\left(300 \mathrm{MHz} \mathrm{CDCl}_{3}\right): \delta \mathrm{ppm}=7.65-7.11$ $(\mathrm{m}, 22 \mathrm{H}), 7.01-6.89$ (m, $2 \mathrm{H},), 6.87$ (d, $J=7.70 \mathrm{~Hz}, 1 \mathrm{H}), 5.40$ (d, $J=4.95 \mathrm{~Hz}, 1 \mathrm{H}), 5.35$ (d, $J$ $=4.70 \mathrm{~Hz}, 1 \mathrm{H}), 5.19(\mathrm{~d}, J=4.70 \mathrm{~Hz}, 1 \mathrm{H}), 5.09(\mathrm{~d}, J=2.69 \mathrm{~Hz}, 2 \mathrm{H}), 4.46(\mathrm{t}, J=7.33,7.33$ $\mathrm{Hz}, 2 \mathrm{H}), 4.20$ (s, $3 \mathrm{H}), 1.93-1.78(\mathrm{~m}, 2 \mathrm{H}), 1.35-1.24(\mathrm{~m}, 2 \mathrm{H}), 0.88$ (t, J = 7.34, $7.34 \mathrm{~Hz}, 3 \mathrm{H})$. ${ }^{13} \mathrm{C}$ NMR $(75 \mathrm{MHz}, C D C l)$ ): $\delta \mathrm{ppm}=156.5\left(\mathrm{O}-\underline{\mathrm{C}}_{\mathrm{ph}}\right), 145.4-113.8\left(\mathrm{~N}-\underline{\mathrm{CH}}=\mathrm{C}, \mathrm{CH}=\underline{\mathrm{C}}-\mathrm{CH}_{2}, \underline{\mathrm{CH}_{\mathrm{ph}}}\right.$

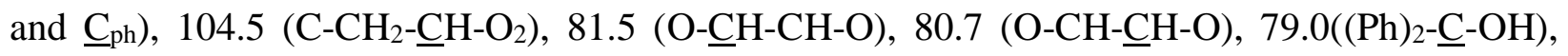

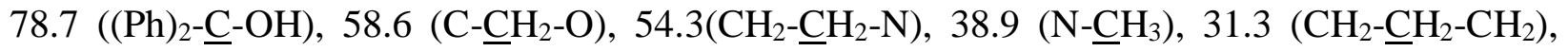
$19.3\left(\mathrm{CH}_{2}-\mathrm{CH}_{2}-\mathrm{CH}_{3}\right), 13.4\left(\mathrm{CH}_{2}-\mathrm{CH}_{2}-\mathrm{CH}_{3}\right)$.

((4R,5R)-4-((3-(4,5-Bis(bis(3,5-dimethylphenyl)(hydroxy)methyl)-1,3-dioxolan-2yl)phenoxy)methyl)-1-butyl-3-methyl-1H-1,2,3-triazol-3-ium tetrafluoroborate

(11b). (4R,5R)-4-((3-(4,5-bis(bis(3,5-dimethylphenyl)(hydroxy)methyl)-1,3-dioxolan-2-

yl)phenoxy)methyl)-1-butyl-3-methyl-1H-1,2,3-triazol-3-ium iodide 10b (922 mg, $1 \mathrm{mmol}$ ) was dissolved in $\mathrm{CH}_{3} \mathrm{CN}$ (2 mL).and to it was added $\mathrm{AgBF}_{4}(195 \mathrm{mg}, 1.0 \mathrm{mmol})$. The reaction mixture was stirred at room temperature for 1 hour. The AgI precipitate was filtered and volatiles were removed from the resulting liquid to furnish the product as a brownish viscous liquid. Yield 99\%. ${ }^{1} \mathrm{H}$ NMR $\left(300 \mathrm{MHz} \mathrm{CDCl}_{3}\right): \delta \mathrm{ppm}=7.43-6.67(\mathrm{~m}, 17 \mathrm{H}), 5.71(\mathrm{~s}, 1 \mathrm{H}), 5.34(\mathrm{~d}, J=4.01$ $\mathrm{Hz}, 1 \mathrm{H}), 5.20(\mathrm{~d}, J=4.24 \mathrm{~Hz}, 1 \mathrm{H}), 5.14(\mathrm{~d}, J=4.48 \mathrm{~Hz}, 2 \mathrm{H}), 4.55(\mathrm{t}, J=7.29,7.29 \mathrm{~Hz}, 2 \mathrm{H})$, $4.22(\mathrm{~s}, 3 \mathrm{H}), 2.36-2.15(\mathrm{~m}, 24 \mathrm{H}), 1.90-1.73(\mathrm{~m}, 2 \mathrm{H}), 1.44-1.28$ (m, $2 \mathrm{H}), 0.95$ (t, J = 7.33, $7.33 \mathrm{~Hz}, 3 \mathrm{H}) .{ }^{13} \mathrm{C} \mathrm{NMR}\left(75 \mathrm{MHz}, \mathrm{CDCl}_{3}\right): \delta \mathrm{ppm}=156.3\left(\mathrm{O}-\underline{\mathrm{C}}_{\mathrm{ph}}\right), 144.7-111.1(\mathrm{~N}-\underline{\mathrm{CH}}=\mathrm{C}$, $\mathrm{CH}=\underline{\mathrm{C}}-\mathrm{CH}_{2}, \underline{\mathrm{CH}_{\mathrm{ph}}}$ and $\left.\underline{\mathrm{C}}_{\mathrm{ph}}\right), 104.6$ (O- $\left.\underline{\mathrm{CH}}-\mathrm{O}\right), 82.0$ (O- $\left.\underline{\mathrm{CH}}-\mathrm{CH}-\mathrm{O}\right), 81.8(\mathrm{O}-\mathrm{CH}-\underline{\mathrm{CH}}-\mathrm{O})$, 80.0((Ph) $\left.)_{2}-\underline{\mathrm{C}}-\mathrm{OH}\right), 78.7\left((\mathrm{Ph})_{2}-\underline{\mathrm{C}}-\mathrm{OH}\right), 61.8\left(\mathrm{C}^{-} \underline{\mathrm{CH}}_{2}-\mathrm{O}\right), 53.6\left(\mathrm{CH}_{2}-\underline{\mathrm{CH}}_{2}-\mathrm{N}\right), 39.7\left(\mathrm{~N}^{-} \underline{\mathrm{CH}}_{3}\right), 31.2$

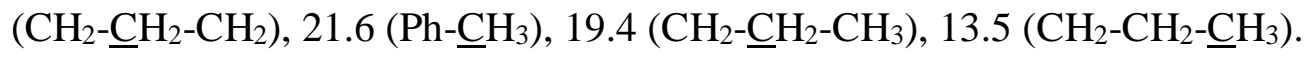

(4R,5R)-4-((3-(4,5-Bis(hydroxydinaphthalen-1-ylmethyl)-1,3-dioxolan-2-yl)phenoxy)-

methyl)-1-butyl-3-methyl-1H-1,2,3-triazol-3-ium tetrafluoroborate (11c). $(4 R, 5 R)-4-((3-(4,5-$ bis(hydroxydinaphthalen-1-ylmethyl)-1,3-dioxolan-2-yl)phenoxy)methyl)-1-butyl-3-methyl-1H1,2,3-triazol-3-ium iodide 10c $(1.1 \mathrm{~g}, 1 \mathrm{mmol})$ was dissolved in $\mathrm{CH}_{3} \mathrm{CN}$ (2 mL) to it was added $\mathrm{AgBF}_{4}(195 \mathrm{mg}, 1.0 \mathrm{mmol})$. The mixture was stirred at room temperature for 1 hour. The AgI precipitate was filtered and volatiles were removed from the resulting liquid to furnish yellowish viscous liquid product. Yield 99\%. ${ }^{1} \mathrm{H}$ NMR (300 $\left.\mathrm{MHz} \mathrm{CDCl}_{3}\right): \delta \mathrm{ppm}=8.95-6.49(\mathrm{~m}, 33 \mathrm{H})$, $6.05(\mathrm{~m}, 1 \mathrm{H}), 5.95(\mathrm{~m}, 1 \mathrm{H}), 5.74(\mathrm{~s}, 1 \mathrm{H}), 4.94(\mathrm{~s}, 2 \mathrm{H}), 4.41(\mathrm{~s}, 3 \mathrm{H}), 4.33$ (t, J = 7.05, 7.05 Hz, $2 \mathrm{H}), 1.90-1.71(\mathrm{~m}, 2 \mathrm{H}), 1.30(\mathrm{dd}, J=14.77,7.33 \mathrm{~Hz}, 2 \mathrm{H}), 0.89$ (t, $J=7.38,7.38 \mathrm{~Hz}, 3 \mathrm{H})$. ${ }^{13} \mathrm{C}$ NMR $\left(75 \mathrm{MHz}, \mathrm{CDCl}_{3}\right): \delta \mathrm{ppm}=159.2\left(\mathrm{O}-\underline{\mathrm{CH}}_{\mathrm{ph}}\right), 144.6-117.4\left(\mathrm{~N}-\underline{\mathrm{C}} \mathrm{H}=\mathrm{C}, \mathrm{C}_{\mathrm{ph}}\right.$ and $\left.\mathrm{C}_{\mathrm{ph}}\right)$,

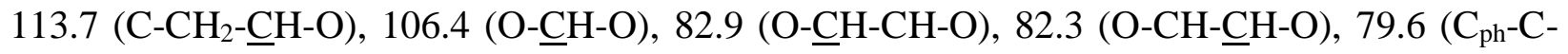


$\mathrm{OH}), 62.1\left(\mathrm{C}-\underline{\mathrm{C}} \mathrm{H}_{2}-\mathrm{O}\right), 49.9\left(\mathrm{CH}_{2}-\underline{\mathrm{CH}}_{2}-\mathrm{N}\right), 38.5\left(\mathrm{~N}-\mathrm{CH}_{3}\right), 32.1\left(\mathrm{CH}_{2}-\underline{\mathrm{CH}}_{2}-\mathrm{CH}_{2}\right), 20.2\left(\mathrm{CH}_{2}-\mathrm{CH}_{2}-\right.$ $\left.\mathrm{CH}_{3}\right), 14.1\left(\mathrm{CH}_{2}-\mathrm{CH}_{2}-\mathrm{CH}_{3}\right)$.

\section{(4R,5R)-1-Benzyl-4-((3-(4,5-bis(hydroxydinaphthalen-1-ylmethyl)-1,3-dioxolan-2-} yl)phenoxy)methyl)-3-methyl-1H-1,2,3-triazol-3-ium tetrafluoroborate (11d). (4R,5R)-1Benzyl-4-((3-(4,5-bis(hydroxydinaphthalen-1-ylmethyl)-1,3-dioxolan-2-yl)phenoxy)methyl)-3methyl-1H-1,2,3-triazol-3-ium iodide 10d (1.0 g, $1 \mathrm{mmol})$ was dissolved in $\mathrm{CH}_{3} \mathrm{CN}$ (2 mL) and to it was added $\mathrm{AgBF}_{4}(195 \mathrm{mg}, 1.0 \mathrm{mmol})$. The mixture was stirred at room temperature for 1 hour. The AgI precipitate was filtered and volatiles were removed from the resulting liquid to furnish the product as a pale yellow viscous liquid. Yield 97\%. ${ }^{1} \mathrm{H} \mathrm{NMR}\left(300 \mathrm{MHz} \mathrm{CDCl}_{3}\right): \delta$ ppm $=8.70-7.15(\mathrm{~m}, 38 \mathrm{H}), 5.61(\mathrm{~s}, 1 \mathrm{H}), 5.53(\mathrm{~s}, 2 \mathrm{H}), 5.35(\mathrm{~d}, J=4.01 \mathrm{~Hz}, 1 \mathrm{H}), 5.36(\mathrm{~d}, J=$ $4.23 \mathrm{~Hz}, 1 \mathrm{H}), 5.13(\mathrm{~d}, J=4.46 \mathrm{~Hz}, 2 \mathrm{H}), 4.0(\mathrm{~s}, 3 \mathrm{H}) .{ }^{13} \mathrm{C} \mathrm{NMR}\left(75 \mathrm{MHz}, \mathrm{CDCl}_{3}\right): \delta \mathrm{ppm}=$ $156.4\left(\mathrm{O}-\underline{\mathrm{C}}_{\mathrm{ph}}\right), 147.9-111.1\left(\mathrm{~N}-\underline{\mathrm{C}} \mathrm{H}=\mathrm{C}, \mathrm{CH}=\underline{\mathrm{C}}-\mathrm{CH}_{2}, \underline{\mathrm{CH}_{\mathrm{ph}}}\right.$ and $\left.\underline{\mathrm{C}}_{\mathrm{ph}}\right), 106.3(\mathrm{O}-\underline{\mathrm{C}} \mathrm{H}-\mathrm{O}), 81.0(\mathrm{O}-$ $\underline{\mathrm{CH}}-\mathrm{CH}-\mathrm{O}), \quad 79.8(\mathrm{O}-\mathrm{CH}-\underline{\mathrm{CH}}-\mathrm{O}), \quad 79.5 \quad\left((\mathrm{Ph})_{2}-\underline{\mathrm{C}}-\mathrm{OH}\right), \quad 78.6\left((\mathrm{Ph})_{2}-\underline{\mathrm{C}}-\mathrm{OH}\right), \quad 61.7\left(\mathrm{C}-\underline{\mathrm{C}} \mathrm{H}_{2}-\mathrm{O}\right)$, 53.5( $\left.\mathrm{Ph}-\underline{\mathrm{CH}}_{2}-\mathrm{N}\right), 37.5\left(\mathrm{~N}-\underline{\mathrm{CH}}_{3}\right)$.

4-(Imidazol-1-yl)butan-2-one (12). ${ }^{29}$ To a stirred solution of imidazole (1.36 g, $\left.20.0 \mathrm{mmol}\right)$ in dry DMSO $(20 \mathrm{~mL})$ was added catalytic amount methylimidazole $(82 \mathrm{mg}, 1.0 \mathrm{mmol})$ and the mixture was cooled to $0{ }^{\circ} \mathrm{C}$. To it was added methyl vinyl ketone $(1.68 \mathrm{~g}, 24.0 \mathrm{mmol})$ and was allowed to warm to room temperature and heated up to $85^{\circ} \mathrm{C}$. When TLC indicated the complete consumption of the starting materials (about three hours) the reaction was made to cool to room temperature. It was diluted with distilled water $(50 \mathrm{~mL})$ and was extracted with ethyl acetate $(50$ $\mathrm{mL})$ three times. The organic layer was separated and washed with additional water $(50 \mathrm{~mL})$. The organic phase was then dried with anhydrous sodium sulphate and volatiles were removed under reduced pressure to furnish the desired product as clear yellow oil. Yield $69 \%$. ${ }^{1} \mathrm{H} \mathrm{NMR}$ $\left(300 \mathrm{MHz} \mathrm{CDCl}_{3}\right): \delta \mathrm{ppm}=7.40(\mathrm{~s}, 1 \mathrm{H}), 6.92(\mathrm{~s}, 1 \mathrm{H}), 6.87(\mathrm{~s}, 1 \mathrm{H}), 4.22-4.05(\mathrm{~m}, 2 \mathrm{H}), 2.84$ $(\mathrm{m}, 2 \mathrm{H}), 2.06(\mathrm{~s}, 3 \mathrm{H}) .{ }^{13} \mathrm{C} \mathrm{NMR}\left(75 \mathrm{MHz}, \mathrm{CDCl}_{3}\right): \delta \mathrm{ppm}=205.1\left(\mathrm{CH}_{3}-\underline{\mathrm{C}}=\mathrm{O}\right), 137.2(\mathrm{~N}=\underline{\mathrm{CH}}-$ $\mathrm{N}), 128.9(\mathrm{~N}-\underline{\mathrm{CH}}=\mathrm{CH}-\mathrm{N}), 119.7(\mathrm{~N}-\underline{\mathrm{CH}}=\mathrm{CH}-\mathrm{N}), 44.3\left(\mathrm{~N}-\underline{\mathrm{CH}_{2}}-\mathrm{CH}_{2}\right), 40.8\left(\mathrm{C}-\underline{\mathrm{CH}}_{2}-\mathrm{CH}_{2}\right), 30.1$ $\left(\mathrm{O}-\mathrm{C}-\underline{\mathrm{CH}}_{3}\right)$.

4-(Imidazol-1-yl)butan-2-one (improved route) (12). To a stirred solution of methyl-vinylketone $(1.40 \mathrm{~g}, 20.0 \mathrm{mmol})$ and imidazole $(1.36 \mathrm{~g}, 20.0 \mathrm{mmol})$ in dry dioxane $(20 \mathrm{~mL})$ was added triethylamine $(0.55 \mathrm{~mL}, 4.0 \mathrm{mmol})$. The reaction mixture refluxed overnight. Volatiles were removed under reduced pressure from the resulting solution. The resulting yellowish oil was subjected to column chromatographic purification with methanol/chloroform gradient 1:19 to furnish 12 in $93 \%$ yield.

((4R,5R)-2-(2-(1H-Imidazol-1-yl)ethyl)-2-methyl-1,3-dioxolane-4,5-dicarboxylate dimethyl ester (13). L-(+)-dimethyl tartrate 4 (1.8 g, $10.1 \mathrm{mmol})$ and 4-(imidazol-1-yl)butan-2-one 12 (920 mg, $6.75 \mathrm{mmol})$ were dissolved in ethyl acetate $(25 \mathrm{~mL})$. The reaction flask was placed in an ice bath to keep the temperature below $20{ }^{\circ} \mathrm{C}$ during the reaction. To the mixture was added $\mathrm{BF}_{3}: \mathrm{OEt}_{2}(3.6 \mathrm{~mL}, 13.5 \mathrm{mmol})$ dropwise. The reaction mixture was left to stir overnight. The resulting solution was poured into saturated sodium bicarbonate solution $(50 \mathrm{~mL})$ and was extracted with EtOAc $(50 \mathrm{~mL})$ three times. The organic layers were combined and washed with 
distilled water $(50 \mathrm{~mL})$ three times and dried over magnesium sulphate. Removal of volatiles under vacuum furnished the desired product as viscous liquid. Yield 78\%. ${ }^{1} \mathrm{H}$ NMR $(300 \mathrm{MHz}$ $\left.\mathrm{CDCl}_{3}\right): \delta \mathrm{ppm}=7.43(\mathrm{~s}, 1 \mathrm{H}), 6.98(\mathrm{~m}, 2 \mathrm{H}), 4.83(\mathrm{~d}, J=5.74 \mathrm{~Hz}, 1 \mathrm{H}), 4.66(\mathrm{~d}, J=5.74 \mathrm{~Hz}, 1$ $\mathrm{H}), 4.19-3.98(\mathrm{~m}, 2 \mathrm{H}), 3.78(\mathrm{~s}, 6 \mathrm{H}), 2.24-2.17(\mathrm{~m}, 2 \mathrm{H}), 1.40(\mathrm{~s}, 3 \mathrm{H}) .{ }^{13} \mathrm{C} \mathrm{NMR}(75 \mathrm{MHz}$, $\left.\mathrm{CDCl}_{3}\right): \delta \mathrm{ppm}=169.8\left(\mathrm{O}=\underline{\mathrm{C}}-\mathrm{OCH}_{3}\right), 169.4\left(\mathrm{O}=\underline{\mathrm{C}}-\mathrm{OCH}_{3}\right), 137.4(\mathrm{~N}=\underline{\mathrm{CH}}-\mathrm{N}), 123.6(\mathrm{~N}-\underline{\mathrm{CH}}=\mathrm{CH}-$ $\mathrm{N}), 121.8(\mathrm{~N}-\mathrm{CH}=\underline{\mathrm{CH}}-\mathrm{N}), 113.3\left(\mathrm{O}-\underline{\mathrm{C}}-\mathrm{CH}_{3}\right), 81.4(\mathrm{O}-\underline{\mathrm{CH}}-\mathrm{CH}), 79.8$ (CH-ㄷH-O), 53.7 (C-O$\left.\underline{\mathrm{CH}}_{3}\right), 52.1\left(\mathrm{C}-\mathrm{O}-\underline{\mathrm{CH}} \mathrm{H}_{3}\right), 44.7\left(\mathrm{~N}-\underline{\mathrm{C}} \mathrm{H}_{2}-\mathrm{CH}_{2}\right), 42.0\left(\mathrm{C}-\underline{\mathrm{C}} \mathrm{H}_{2}-\mathrm{CH}_{2}\right), 24.6\left(\mathrm{O}_{2}-\mathrm{C}-\underline{\mathrm{C}} \mathrm{H}_{3}\right) .[\alpha]_{D}^{28}=-14.5^{\circ}$ $\left(c=1, \mathrm{CHCl}_{3}\right)$.

((4R,5R)-2-(2-(1H-Imidazol-1-yl)ethyl)-2-methyl-1,3-dioxolane-4,5-diyl)bis(diphenyl-

methanol) (14). A reaction flask fitted with an efficient condenser was dried with hot air-gun and flushed with argon 3 times. Grignard reagent was prepared from magnesium turnings (243 $\mathrm{mg}, 10 \mathrm{mmol})$ in dry THF $(15 \mathrm{~mL})$ with a slow addition of bromobenzene $(1.9 \mathrm{~g}, 12 \mathrm{mmol})$. Initially the reaction mixture was set to reflux by itself with a controlled addition of the bromobenzene. After the complete consumption of the $\mathrm{Mg}$ tunings refluxing was continued for 1 h using a water-bath. 2-(2-(1H-imidazol-1-yl)ethyl)-2-methyl-1,3-dioxolane-4,5-dicarboxylate dimethyl ester 13 (298 mg, $1 \mathrm{mmol})$ in dry THF (2 mL) was slowly added to the Grignard reagent at room temperature. The reaction mixture was left to stir overnight. The resulting solution was diluted with $\mathrm{Et}_{2} \mathrm{O}(20 \mathrm{~mL})$ and quenched by $1 \mathrm{~N} \mathrm{aq} \mathrm{HCl}(50 \mathrm{~mL})$ at $0{ }^{\circ} \mathrm{C}$. The ether phase was separated and dried with $\mathrm{MgSO}_{4}$ and the solvent was removed under vacuum to give the title product as yellowish solid. Yield 58\%. M.p. $103-105{ }^{\circ} \mathrm{C}$. ${ }^{1} \mathrm{H} \mathrm{NMR}(300 \mathrm{MHz} \mathrm{CDCl})$ : $\delta \mathrm{ppm}=8.58(\mathrm{~s}, 1 \mathrm{H}), 7.43-7.22(\mathrm{~m}, 20 \mathrm{H}), 7.11(\mathrm{~m}, 1 \mathrm{H}), 6.65(\mathrm{~m}, 1 \mathrm{H}), 4.74(\mathrm{~d}, J=7.95 \mathrm{~Hz}$, $1 \mathrm{H}), 4.56$ (d, $J=7.98 \mathrm{~Hz}, 1 \mathrm{H}), 3.76(\mathrm{~m}, 1 \mathrm{H}), 3.59(\mathrm{~m}, 1 \mathrm{H}), 1.93(\mathrm{~m}, 1 \mathrm{H}), 1.82(\mathrm{~m}, 1 \mathrm{H}), 0.90$ $(\mathrm{s}, 3 \mathrm{H}) .{ }^{13} \mathrm{C} \mathrm{NMR}\left(75 \mathrm{MHz}, \mathrm{CDCl}_{3}\right): \delta \mathrm{ppm}=146.0-142.1\left(\mathrm{HO}-\mathrm{C}-\underline{\mathrm{C}}_{\mathrm{ph}}\right), 134.6(\mathrm{~N}=\underline{\mathrm{CH}}-\mathrm{N})$,

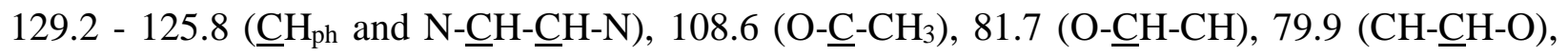
$78.1\left((\mathrm{Ph})_{2}-\underline{\mathrm{C}}-\mathrm{OH}\right), 78.1\left((\mathrm{Ph})_{2}-\underline{\mathrm{C}}-\mathrm{OH}\right), 44.1\left(\mathrm{~N}-\underline{\mathrm{CH}}_{2}-\mathrm{CH}_{2}\right), 39.8\left(\mathrm{C}-\underline{\mathrm{CH}}_{2}-\mathrm{CH}_{2}\right), 25.6\left(\mathrm{O}_{2}-\mathrm{C}-\underline{\mathrm{C}} \mathrm{H}_{3}\right)$. $[\alpha]_{D}^{28}=-23.1^{\circ}\left(c=1, \mathrm{CHCl}_{3}\right)$, HRMS (ESI): Found: $m / z$ 545.2446. calculated for $\mathrm{C}_{35} \mathrm{H}_{33} \mathrm{~N}_{2} \mathrm{O}_{4}$ $\left(\mathrm{M}-\mathrm{H}^{+}\right): 545.2440$.

(4R,5R)-1-(2-(4,5-Bis(hydroxydiphenylmethyl)-2-methyl-1,3-dioxolan-2-yl)ethyl)-3-methyl$\mathbf{1 H}$-imidazol-3-ium iodide (15). A stirred solution of TADDOL 14 (5.43 g, $10.0 \mathrm{mmol}$ ) was dissolved in acetonitrile $(100 \mathrm{~mL})$ in a sealed two necked flask fitted with an efficient reflux condenser and an argon filled balloon. Methyl iodide (7 g, $50 \mathrm{mmol}$ ) was injected dropwise. The reaction mixture was refluxed overnight. From the resulting yellowish solution, volatiles were removed with a rotary evaporator under a well-ventilated hood to give the title product as a brown thick liquid. Yield 95\%. ${ }^{1} \mathrm{H}$ NMR $\left(300 \mathrm{MHz} \mathrm{CDCl}_{3}\right): \delta \mathrm{ppm}=8.91(\mathrm{~s}, 1 \mathrm{H}), 7.43-7.12$ $(\mathrm{m}, 20 \mathrm{H}), 6.92-6.84(\mathrm{~m}, 2 \mathrm{H}), 4.67$ (d, $J=7.85 \mathrm{~Hz}, 1 \mathrm{H}), 4.48(\mathrm{~d}, J=7.85 \mathrm{~Hz}, 1 \mathrm{H}), 3.76(\mathrm{~s}, 3$ $\mathrm{H}), 3.72-3.53(\mathrm{~m}, 2 \mathrm{H}), 2.16-1.98(\mathrm{~m}, 1 \mathrm{H}), 1.92-1.75(\mathrm{~m}, 1 \mathrm{H}), 0.87(\mathrm{~s}, 3 \mathrm{H}) .{ }^{13} \mathrm{C} \mathrm{NMR}(75$ $\left.\mathrm{MHz}, \mathrm{CDCl}_{3}\right): \delta \mathrm{ppm}=145.6-141.9\left(\mathrm{HO}-\mathrm{C}-\underline{\mathrm{C}}_{\mathrm{ph}}\right), 135.8(\mathrm{~N}=\underline{\mathrm{C}} \mathrm{H}-\mathrm{N}), 129.0-127.4\left(\underline{\mathrm{CH}}_{\mathrm{ph}}\right), 123.8$ and $122.1(\mathrm{~N}-\underline{\mathrm{C}} \mathrm{H}=\underline{\mathrm{C}} \mathrm{H}-\mathrm{N}), 108.7\left(\mathrm{O}-\underline{\mathrm{C}}-\mathrm{CH}_{3}\right), 81.7(\mathrm{O}-\underline{\mathrm{CH}}-\mathrm{CH}), 80.0(\mathrm{CH}-\underline{\mathrm{CH}}-\mathrm{O}), 78.2\left((\mathrm{Ph})_{2}-\underline{\mathrm{C}}-\right.$ $\mathrm{OH}), 78.1\left((\mathrm{Ph})_{2}-\underline{\mathrm{C}}-\mathrm{OH}\right), 45.0\left(\mathrm{~N}-\underline{\mathrm{CH}}_{2}-\mathrm{CH}_{2}\right), 39.5\left(\mathrm{C}-\underline{\mathrm{C}} \mathrm{H}_{2}-\mathrm{CH}_{2}\right), 37.1\left(\mathrm{~N}-\underline{\mathrm{C}} \mathrm{H}_{3}\right), 25.6\left(\mathrm{O}_{2}-\mathrm{C}-\underline{\mathrm{C}} \mathrm{H}_{3}\right)$. 
$[\alpha]_{D}^{28}=-15.8\left(c=1, \mathrm{CHCl}_{3}\right)$. HRMS (ESI): Found: $m / z$ 561.2748. Calculated for $\mathrm{C}_{21} \mathrm{H}_{26} \mathrm{~N}_{3} \mathrm{O}_{3} \mathrm{~S}$ $\left(\mathrm{M}^{+}\right): 561.2748$.

(4R,5R)-1-(2-(4,5-Bis(hydroxydiphenylmethyl)-2-methyl-1,3-dioxolan-2-yl)ethyl)-3-methyl$1 H$-imidazol-3-ium tetrafluoroborate (16). To a solution of $(4 R, 5 R)-1-(2-(4,5-$ bis(hydroxydiphenylmethyl)-2-methyl-1,3-dioxolan-2-yl)ethyl)-3-methyl-1H-imidazol-3-ium iodide 15 (690 mg, $1 \mathrm{mmol}$ ) in $\mathrm{CH}_{3} \mathrm{CN}(2 \mathrm{~mL}) \mathrm{AgBF}_{4}(195 \mathrm{mg}, 1.0 \mathrm{mmol})$ was added. The reaction mixture was stirred at room temperature for one hour. The solid precipitate (AgI) was filtered off and volatiles were removed from the filtrate to furnish the title product as a brown viscous liquid. Yield $99 \%$. ${ }^{1} \mathrm{H}$ NMR $\left(300 \mathrm{MHz} \mathrm{CDCl}_{3}\right): \delta \mathrm{ppm}=8.90(1 \mathrm{H}, \mathrm{s}, \mathrm{N}=\mathrm{C} \underline{\mathrm{H}}-\mathrm{N}), 7.39$ $7.19(\mathrm{~m}, 20 \mathrm{H}), 6.93-6.93(\mathrm{~m}, 2 \mathrm{H}), 4.77(\mathrm{~d}, J=7.85 \mathrm{~Hz}, 1 \mathrm{H}), 4.50(\mathrm{~d}, J=7.85 \mathrm{~Hz}, 1 \mathrm{H}), 3.76$ (s, $3 \mathrm{H}), 3.70$ - 3.49 (m, $2 \mathrm{H}), 2.16-1.98(\mathrm{~m}, 1 \mathrm{H}), 1.92$ - $1.75(\mathrm{~m}, 1 \mathrm{H}), 0.87$ (s, $3 \mathrm{H}) .{ }^{13} \mathrm{C} \mathrm{NMR}$ $\left(75 \mathrm{MHz}, \mathrm{CDCl}_{3}\right): \delta \mathrm{ppm}=145.8-141.9\left(\mathrm{HO}-\mathrm{C}-\underline{\mathrm{C}}_{\mathrm{ph}}\right), 136.1(\mathrm{~N}=\underline{\mathrm{CH}}-\mathrm{N}), 129.0-126.9\left(\underline{\mathrm{CH}}_{\mathrm{ph}}\right)$,

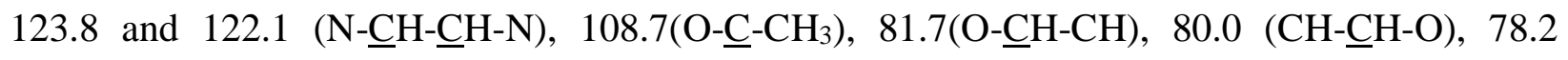
$\left.(\mathrm{Ph})_{2}-\underline{\mathrm{C}}-\mathrm{OH}\right), 78.1\left((\mathrm{Ph})_{2}-\underline{\mathrm{C}}-\mathrm{OH}\right), 45.0\left(\mathrm{~N}-\underline{\mathrm{CH}}_{2}-\mathrm{CH}_{2}\right), 39.5\left(\mathrm{C}-\underline{\mathrm{C}} \mathrm{H}_{2}-\mathrm{CH}_{2}\right), 37.2\left(\mathrm{~N}_{-} \underline{\mathrm{CH}}_{3}\right), 25.6\left(\mathrm{O}_{2}-\right.$ C- $\left.-\mathrm{CH}_{3}\right)$.

4-Methoxy-6-phenyl-5,6-dihydro-2H-pyran-2-one (18). ${ }^{30}$ The TADDOL catalyst $11 d$ (97 mg, $0.1 \mathrm{mmol}$ ) was placed in a small reaction vial. The air was evacuated at 10 mbar and was flushed with argon three times. A freshly distilled benzaldehyde $(256 \mathrm{mg}, 2.5 \mathrm{mmol})$ was then injected and the contents were cooled to $-24^{\circ} \mathrm{C}$. Brassard's diene 17 (102 mg, $\left.0.5 \mathrm{mmol}\right)$ was added and the mixture was stirred at $-24{ }^{\circ} \mathrm{C}$. At the completion of the reaction $\mathrm{MeOH}(0.5 \mathrm{~mL})$ was added and stirred for $30 \mathrm{~min}$ at the same temperature. Volatiles were solvent was evaporated and the remainder was extracted with $\mathrm{Et}_{2} \mathrm{O}(3 \mathrm{~mL}) 3$ times. The $\mathrm{Et}_{2} \mathrm{O}$ phase was removed to furnish the title product after column chromatography (Cyclohexane: Ethyl acetate, $2: 1$ ). Yield 49\%. ${ }^{1} \mathrm{H}$ NMR (300 MHz CDCl $): \delta$ ppm = $7.41-7.19(\mathrm{~m}, 5 \mathrm{H}), 5.47(\mathrm{~m}, 1 \mathrm{H}), 5.31(\mathrm{~s}, 1 \mathrm{H}), 3.78(\mathrm{~s}, 3$ $\mathrm{H}), 2.85-2.88(\mathrm{~m}, 1 \mathrm{H}), 2.64-2.56(\mathrm{~m}, 1 \mathrm{H}) .{ }^{13} \mathrm{C} \mathrm{NMR}\left(75 \mathrm{MHz}, C D C l_{3}\right): \delta \mathrm{ppm}=171.3$ $(\mathrm{CH}=\underline{\mathrm{C}}-\mathrm{O}), 167.4(\mathrm{O}-\underline{\mathrm{C}}=\mathrm{O}), 139.6\left(\mathrm{C}_{\mathrm{ph}} \underline{\mathrm{C}} \mathrm{H}-\mathrm{O}\right), 129.9$ - $124.4\left(\underline{\mathrm{CH}_{\mathrm{ph}}}\right), 89.4(\mathrm{C}=\underline{\mathrm{CH}}-\mathrm{C}), 76.9(\mathrm{O}-$ $\left.\underline{\mathrm{CH}}-\mathrm{CH}_{2}\right), 56.8\left(\underline{\mathrm{CH}}_{3}-\mathrm{O}\right), 35.1\left(\mathrm{C}-\underline{\mathrm{CH}}_{2}-\mathrm{CH}\right)$. Enantioselectivity $=12 \%$ (HPLC on a Chiralpak $\mathrm{AD}$ column, $\lambda=215 \mathrm{~nm}$, Eluent $i-\mathrm{PrOH}$-hexane $(85: 15)$, flow rate $=1.0 \mathrm{~mL} \mathrm{~min}^{-} 1 ; t \mathrm{R}=16.31$ min (minor), 18.06 min (major)).

2-Phenyl-2H-pyran-4(3H)-one (20). ${ }^{35}$ Rawal's diene 19 (114 mg, $\left.0.5 \mathrm{mmol}\right)$ was injected into a solution of catalyst 11d (97 mg $0.1 \mathrm{mmol}$ ) and freshly distilled benzaldehyde (530 mg, $5 \mathrm{mmol}$ ) in a sealed reaction flask under argon atmosphere at $-78{ }^{\circ} \mathrm{C}$. After 48 hours at the same temperature dry $\mathrm{Et}_{2} \mathrm{O}(2 \mathrm{~mL})$ was injected to separate the reaction mixture from the IL-tagged catalyst by precipitation, the $\mathrm{Et}_{2} \mathrm{O}$ phase was subjected to acetyl chloride $352 \mu \mathrm{L}$ at $-78{ }^{\circ} \mathrm{C}$. The mixture was then stirred for half hour and volatiles were removed. The residue was purified by column chromatography (Cyclohexane : EtOAc, 3:1) to furnish the title product. Yield $32 \% .{ }^{1} \mathrm{H}$ NMR $\left(300 \mathrm{MHz}, \mathrm{CDCl}_{3}\right): \delta \mathrm{ppm}=7.67-7.43(\mathrm{~m}, 5 \mathrm{H}), 7.31(\mathrm{~s}, 1 \mathrm{H}), 5.56(\mathrm{~d}, J=6.02,1 \mathrm{H})$, $5.45(\mathrm{dd}, J=14.39,3.51 \mathrm{~Hz}, 1 \mathrm{H}), 2.94-2.88(\mathrm{~m}, 1 \mathrm{H}), 2.69-2.56(\mathrm{~m}, 1 \mathrm{H}) .{ }^{13} \mathrm{C}$ NMR $(75$ $\left.\mathrm{MHz}, \mathrm{CDCl}_{3}\right): \delta \mathrm{ppm}=195.2(\mathrm{CH}-\underline{\mathrm{C}}=\mathrm{O}), 163.4(\mathrm{O}-\underline{\mathrm{C}} \mathrm{H}=\mathrm{CH}), 131.6-126.1\left(\underline{\mathrm{CH}}_{\mathrm{ph}}, \underline{\mathrm{C}}_{\mathrm{ph}}\right), 107.4$ $(\mathrm{C}-\underline{\mathrm{C}} \mathrm{H}=\mathrm{CH}), 89.4$ (Ph- $\underline{\mathrm{CH}}-\mathrm{O}), 43.4\left(\mathrm{C}-\underline{\mathrm{CH}}_{2}-\mathrm{CH}\right)$. Enantioselectivity $=26 \%$ (HPLC on a 
Chiralpak OD column, $\lambda=215 \mathrm{~nm}$, Eluent $i$-PrOH-hexane $(9: 1)$, flow rate $=0.5 \mathrm{~mL}$ min $^{-} 1 ; t \mathrm{R}=$ $34.4 \mathrm{~min}$ (major), $40.3 \mathrm{~min}$ (minor).

\section{Acknowledgements}

We gratefully acknowledge financial support by Deutsche Forschungsgemeinschaft (DFG, priority program “Organocatalysis” SPP1179). Yacob. Z. is indebted to Deutscher Akademischer Austauschdienst (DAAD) for a study grant.

\section{References}

1. Seebach, D.; Beck, A. K.; Schiess, M.; Widler, L.; Wonnacott, A. Pure Appl Chem 1983, 55, 1807.

2. Seebach, D.; Beck, A. K.; Heckel, A. Angew Chem Int Ed 2001, 40, 92.

3. Altava, B.; Burguete, M. I.; Escuder, B.; Luis, S. V.; Salvador, R. V.; Fraile, J. M.; Mayoral, J. A.; Royo, A. J. J Org Chem 1997, 62, 3126.

4. Altava, B.; Burguete, M. I.; Garcia-Verdugo, E.; Luis, S. V.; Vicent, M. J. Green Chem 2006, 8, 717.

5. Atwood, J. L.; MacNicol, D.; Toda, F.; Bishop, R. Comprehensive supramolecular chemistry. Vol. 6, Solid-state supramolecular chemistry: crystal engineering; Pergamon: New York, 1996.

6. Luo, S.; Mi, X.; Zhang, L.; Liu, S.; Xu, H.; Cheng, J. P. Angew Chem Int Ed 2006, 45, 3093.

7. Luo, S. P.; Xu, D. Q.; Yue, H. D.; Wang, L. P.; Yang, W. L.; Xu, Z. Y. Tetrahedron:Asymmetry 2006, 17, 2028.

8. Yan, Z. Y.; Niu, Y. N.; Wei, H. L.; Wu, L. Y.; Zhao, Y. B.; Liang, Y. M. Tetrahedron:Asymmetry 2006, 17, 3288.

9. Yacob, Z.; Shah, J.; Leistner, J.; Liebscher, J. Synlett 2008, 2342.

10. Ding, X.; Tang, W. M.; Zhu, C. J.; Cheng, Y. X. Adv Synth Catal 2010, 352, 108.

11. Sebesta, R.; Kmentova, I.; Toma, S. Green Chem 2008, 10, 484.

12. Maltsev, O. V.; Chizhov, A. O.; Zlotin, S. G. Chem-Eur J 2011, 17, 6109.

13. Shah, J.; Khan, S. S.; Blumenthal, H.; Liebscher, J. Synthesis 2009, 3975.

14. Maltsev, O. V.; Kucherenko, A. S.; Beletskaya, I. P.; Tartakovsky, V. A.; Zlotin, S. G. Eur J Org Chem 2010, 2927.

15. Yacob, Z.; Liebscher, J. In Ionic Liquids - Classes and Properties; Handy, S. T. Ed.; InTech: Rijeka, Croatia, 2011; pp. 3

16. Parvulescu, V. I.; Hardacre, C. Chem Rev 2007, 107, 2615.

17. Lombardo, M.; Pasi, F.; Easwar, S.; Trombini, C. Adv Synth Catal 2007, 349, 2061.

18. Zhou, L.; Wang, L. Chem Lett 2007, 36, 628. 
19. Maltsev, O. V.; Kucherenko, A. S.; Zlotin, S. G. Mendeleev Commun 2011, 21, 146.

20. Khan, S. S.; Hanelt, S.; Liebscher, J. Arkivoc 2009, (xii), 193.

21. Khan, S. S.; Shah, J.; Liebscher, J. Tetrahedron 2010, 66, 9468.

22. Khan, S. S.; Shah, J.; Liebscher, J. Tetrahedron 2011, 67, 1812

23. Kolb, H. C.; Finn, M. G.; Sharpless, K. B. Angew Chem Int Ed 2001, 40, 2004.

24. Hanelt, S.; Liebscher, J. Synlett 2008, 1058.

25. Seebach, D.; Pichota, A.; Beck, A. K.; Pinkerton, A. B.; Litz, T.; Karjalainen, J.; Gramlich, V. Org Lett 1999, 1, 55.

26. Bateman, S. A.; Kelly, D. P.; Martin, R. F.; White, J. M. Aust J Chem 1999, 52, 291.

27. Chibale, K.; Hans, R. H.; Guantai, E. M.; Lategan, C.; Smith, P. J.; Wan, B. J.; Franzblau, S. G.; Gut, J.; Rosenthal, P. J. Bioorg Med Chem Lett 2010, 20, 942.

28. Smith, M.; Khorana, H. G.; Rammler, D. H.; Goldberg, I. H. J Am Chem Soc 1962, 84, 430.

29. Liu, B. K.; Wu, Q.; Qian, X. Q.; Lv, D. S.; Lin, X. F. Synthesis 2007, 2653.

30. Du, H. F.; Zhao, D. B.; Ding, K. L. Chem-Eur J 2004, 10, 5964.

31. Narasaka, K.; Inoue, M.; Yamada, T.; Sugimori, J.; Iwasawa, N. Chem Lett 1987, 2409.

32. Huang, Y.; Rawal, V. H. J Am Chem Soc 2002, 124, 9662.

33. Huang, Y.; Rawal, V. H. Org Lett 2000, 2, 3321.

34. Huang, Y.; Unni, A. K.; Thadani, A. N.; Rawal, V. H. Nature 2003, 424, 146.

35. Hashimoto, S.; Watanabe, Y.; Washio, T.; Shimada, N.; Anada, M. Chem Commun 2009, 7294. 